The Astrophysical Journal, 676:1262-1280, 2008 April 1

(C) 2008. The American Astronomical Society. All rights reserved. Printed in U.S.A.

\title{
SIMULATIONS OF DYNAMO ACTION IN FULLY CONVECTIVE STARS
}

\author{
Matthew K. Browning ${ }^{1}$ \\ Astronomy Department, 601 Campbell Hall, University of California, Berkeley, CA 94720-3411 \\ Received 2007 October 9; accepted 2007 December 3
}

\begin{abstract}
We present three-dimensional nonlinear magnetohydrodynamic simulations of the interiors of fully convective M dwarfs. Our models consider 0.3 solar-mass stars using the Anelastic Spherical Harmonic code, with the spherical computational domain extending from 0.08 to 0.96 times the overall stellar radius. Like previous authors, we find that fully convective stars can generate kG-strength magnetic fields (in rough equipartition with the convective flows) without the aid of a tachocline of shear. Although our model stars are everywhere unstably stratified, the amplitudes and typical pattern sizes of the convective flows vary strongly with radius, with the outer regions of the stars hosting vigorous convection and field amplification while the deep interiors are more quiescent. Modest differential rotation is established in hydrodynamic calculations, but - unlike in some prior work - strongly quenched in MHD simulations because of the Maxwell stresses exerted by the dynamo-generated magnetic fields. Despite the lack of strong differential rotation, the magnetic fields realized in the simulations possess significant mean (axisymmetric) components, which we attribute partly to the strong influence of rotation on the slowly overturning flows.

Subject headings: convection - MHD — stars: low-mass, brown dwarfs — stars: magnetic fields — turbulence
\end{abstract}

\section{INTRODUCTION}

Magnetic fields are ubiquitous in stars. Across a wide range of stellar masses, these fields are thought to arise from dynamo action, with convection, rotation, and shear all likely players in the process of field generation (e.g., Moffatt 1978). Stars whose interiors are everywhere convectively unstable have been thought to harbor dynamos that may differ fundamentally from those in stars that also possess radiative cores (e.g., Durney et al. 1993). Recent observations and theoretical models are greatly complicating this basic view, and a thorough understanding of the dynamo process in such stars remains elusive. We begin here by outlining both the current theoretical understanding of the magnetism of stars with and without internal stable zones, and some recent observational puzzles that serve to motivate our work.

\subsection{Magnetic Fields in Solar-Type Stars}

In the Sun, the boundary layer between the convective envelope and the stably stratified core is believed to play a pivotal role in the generation of global magnetic fields by dynamo action (e.g., Ossendrijver 2003). Helioseismology has revealed that this interface region is a site of strong shear, where the solar angular velocity profile transitions from differential rotation - with a fast equator, slow poles, and angular velocity nearly constant on radial lines within the convection zone - to solid-body rotation within the radiative core (e.g., Thompson et al. 1996). In the standard "interface dynamo" paradigm for the global solar dynamo, this shearing layer, called the tachocline, stretches and amplifies poloidal magnetic fields generated within the convection zone, giving rise to organized toroidal field structures (e.g., Parker 1993; Charbonneau \& MacGregor 1997; Ossendrijver 2003). These toroidal fields may ultimately become unstable to magnetic buoyancy instabilities and rise through the convection zone, with some eventually appearing at the surface as sunspots and others being shredded by the convection and used to create

\footnotetext{
${ }^{1}$ Current address: Department of Astronomy and Astrophysics, University of Chicago, 5640 South Ellis Avenue, Chicago, IL 60637.
}

poloidal fields, thereby completing the dynamo cycle. Alternatively, it has been argued that coherent meridional circulations may bring fields to the surface (e.g., Rempel 2006; Dikpati \& Charbonneau 1999). In either case, the tachocline is likely a key element in the solar dynamo process - partly because it is a site of strong shear, but also because the stable stratification that prevails below the convection zone allows fields to be greatly amplified before they become unstable to magnetic buoyancy instabilities and rise. In the convection zone, by contrast, the timescale for field amplification to the $\sim 2000-3000 \mathrm{G}$ commonly observed in sunspots (e.g., Simon et al. 1988; Stix 2002) is longer than simple estimates of the timescale for flux tubes to rise due to this instability (Parker 1975).

Simulations and mean-field models have helped to affirm the likely importance of the tachocline in generating the large-scale magnetism of stars like the Sun. Three-dimensional magnetohydrodynamic (MHD) simulations that modeled convection zones in various geometries have demonstrated that helical convection can readily build strong $(\mathrm{kG})$ magnetic fields; in some cases (e.g., Jones \& Roberts 2000; Stillmach \& Hansen 2004), both small- and large-scale fields were realized. However, several recent calculations of turbulent flows (at finite Prandtl number) have suggested that generating a large-scale field may be quite difficult unless the flow is highly organized (see discussion in Cattaneo \& Hughes 2006); other authors have argued that boundary conditions may play a crucial role in allowing large-scale field generation (e.g., Blackman \& Field 2000). Recent 3D spherical shell simulations that modeled the bulk of the solar convective envelope (Brun et al. 2004, hereafter BMT04) have lent some support to the view that convection alone may have difficulty in building solar-like magnetism: dynamo action was realized, but the fields tended to be mostly on small spatial scales, exhibited no evident parity preferences, and showed a tendency to reverse in polarity on irregular intervals of only a few hundred days. Building on this work, recent simulations also included penetration by the turbulent convection into a stable region with an imposed tachocline of shear (Browning et al. 2006). These calculations showed that large-scale toroidal fields could indeed be realized within the tachocline; 
furthermore, these fields possessed antisymmetric parity like that observed in sunspots, and showed much more stable polarity than in simulations of the convection zone alone. Likewise, extensive mean-field modeling has generally also suggested that differential rotation in the tachocline plays a dominant role in generating toroidal fields from poloidal fields, a process parameterized as the $\Omega$-effect (e.g., Moffatt 1978; Steenbeck et al. 1966). In the Sun, this is taken to act in concert with the $\alpha$-effect that can produce a toroidal field from a poloidal field (and vice versa), either through the action of cyclonic convection or through BabcockLeighton effects near the surface (e.g., Parker 1955; Steenbeck et al. 1966; Babcock 1961; Leighton 1969). Together, these constitute the solar $\alpha-\Omega$ dynamo.

\subsection{Puzzles of M Star Magnetism}

Stars less massive than about 0.35 solar masses are fully convective, and so cannot possess a transition region precisely like the solar tachocline. It therefore seems natural to expect that they should harbor qualitatively different magnetic dynamo action than stars like the Sun (e.g., Durney et al. 1993). Yet no obvious transition in magnetic activity has been observed at spectral types $\sim \mathrm{M} 3$, where the stably stratified core disappears. Instead, stars on both sides of this "tachocline divide" appear to be able to build magnetic fields effectively. Many fully convective stars are observed to have strong chromospheric $\mathrm{H} \alpha$ emission (e.g., Hawley et al. 1996; Mohanty \& Basri 2003; West et al. 2004), which is well established as an indication of the presence of magnetic fields. Indeed, the fraction of stars that show such emission increases markedly after the transition to full convection, reaching a maximum around spectral type M8 (West et al. 2004). Magnetic fields have also recently been directly detected on fully convective stars using magnetically sensitive FeH line ratios (Reiners \& Basri 2007).

It remains unclear whether the magnetic fields in such stars differ fundamentally - either in spatial structure, temporal variability, or dependence on stellar parameters like rotation ratefrom those in more massive stars. In stars with spectral types ranging from mid-F to early $\mathrm{M}$, observations indicate that chromospheric and coronal activity increase rapidly with increasing rotational velocity, then saturate above a threshold velocity (e.g., Noyes et al. 1984; Pizzolato et al. 2003; Delfosse et al. 1998). This threshold velocity appears to lessen with decreasing stellar mass. Some evidence also exists for a "supersaturation" regime, with magnetic activity somewhat lessened in the most rapid rotators (e.g., James et al. 2000). The situation is less clear in the mid- to late-M dwarfs. Mohanty \& Basri (2003) argued that a sample of stars ranging from M4 to M9 exhibited a common "saturation type" rotation-activity relationship, with observed activity roughly independent of rotation rate above a threshold value. Measuring the rotation rates of the slowest rotators is difficult, so it remains unclear whether magnetic activity in these stars increases gradually with rotation as in solar-like stars, or instead changes more abruptly. Very recently, two fully convective M6 stars have been found with reasonably rapid rotation $(v \sin i>$ $5 \mathrm{~km} \mathrm{~s}^{-1}$ ) but no measurable chromospheric emission (A. West \& G. Basri 2008, in preparation): these stars are not so cool that low conductivity effects are likely to play a major role (see Mohanty et al. 2002), so these observations raise further questions about how the magnetic fields of such stars are influenced by rotation. Another striking constraint on the strength and morphology of $\mathrm{M}$ dwarf magnetic fields has been provided by Donati et al. (2006), who used Zeeman Doppler imaging to show that the rapidly rotating fully convective star v374 Peg possesses a large- scale axisymmetric field of $\mathrm{kG}$ strength, but no surface differential rotation.

An additional complication is that the rotation rates of stars (and hence perhaps their magnetic activity) depend strongly on their age: stars generally arrive on the main sequence rapidly rotating, and slow over time through angular momentum loss via a magnetized wind (e.g., Skumanich 1972; Weber \& Davis 1967). The amount of rotational braking a star undergoes at any point in its evolution is then presumably dependent on the strength of its magnetic field, and perhaps also on the geometry of that field. Thus, differences between the dynamo acting on either side of the "tachocline divide" might manifest themselves as differences in the rotational evolution of stars with or without radiative cores. Indeed, some recent evidence suggests that the timescale for magnetic braking, as indicated by the typical ages at which stars are no longer observably rotating, may increase markedly at approximately the mass where full convection sets in (e.g., West et al. 2007; Reiners et al. 2007; Barnes 2003).

\subsection{Prior Modeling and this Work}

A few previous authors have examined the generation of magnetic fields in fully convective stars using either semianalytical theory or simulation, but the overall picture that emerges from these investigations is somewhat murky. Durney et al. (1993) argued that in the absence of a tachocline of shear, the magnetic fields of fully convective stars should be dominated by smallscale dynamo action, with typical spatial scales of order the size of convective cells. Küker \& Rüdiger (1999) examined dynamos in fully convective pre-main-sequence stars using mean field theory; they assumed that such stars rotate approximately as rigid bodies, and adopted a simple $\alpha$-quenching formula to account crudely for the back-reaction of dynamo-generated fields on the flows. They found that $\alpha^{2}$ dynamo solutions could be excited for moderate rotation rates, giving rise to steady, nonaxisymmetric mean fields. Chabrier \& Küker (2006) performed analogous mean field modeling for fully convective low-mass stars, typically also assuming no differential rotation, and found large-scale nonaxisymmetric fields were generated by an $\alpha^{2}$ dynamo; these fields were steady and symmetric with respect to the equatorial plane. Chabrier \& Küker (2006) also constructed a model with internal differential rotation (which they argued might apply to brown dwarfs with conductive cores), and found that predominantly toroidal axisymmetric fields were generated by the $\alpha^{2}-\Omega$ dynamo that resulted. Finally, Dobler et al. (2006, hereafter DSB06) conducted 3D hydrodynamic and MHD simulations of fully convective spheres using a Cartesian grid-based finitedifference code. They found that such stars established "antisolar" differential rotation, with the poles rotating more rapidly than the equator. Dynamo action was realized, ultimately yielding typical magnetic field strengths approximately in equipartition with the flows near the surface. The resulting magnetic fields possessed structure on a range of spatial scales, with a substantial large-scale component.

The origins of the discrepancies between these theoretical predictions are unclear. Part of the difficulty lies in the inherent limitations of mean field modeling, which separates the flows and fields into a large-scale (mean) component and everything else, with the latter typically parameterized in terms of the mean fields using a turbulence closure model. Such modeling cannot provide detailed descriptions of the spatial distribution of the magnetic fields; nor can it independently constrain the field strengths that are achieved by dynamo action, since the models usually adopt $\alpha$-quenching prescriptions that simply eliminate 
the $\alpha$-effect as equipartition is reached. Furthermore, the differential rotation realized by the convection was essentially a free parameter within the models quoted above. Nonetheless, the results of such modeling are highly suggestive of the roles played by convection and rotation in building magnetic fields on both large and small scales. Indeed, a variety of related dynamo-theoretic work, in particular calculations of MHD spectra within the eddydamped quasi-normal Markovian approximation (Pouquet et al. 1976) indicates that convection with helicity — as imparted by rotation - may lead to cascades of magnetic energy from small toward large scales, again hinting that shear need not be present in order to generate large-scale magnetic fields. Meanwhile, the results of the numerical simulations thus far cannot be taken as the last word on the subject either: although they provide descriptions of the dynamics on many different scales, they are still limited by numerical resolution to parameter regimes far removed from those of stellar convection. Furthermore, it is difficult to gauge a priori how the many different simplifications adopted within DSB06 (or any other simulation) might impact their results. In any event, the conflict between the results of all theoretical models published so far and the observational constraints on field geometry provided by Donati et al. (2006) lend further vibrancy to the study of magnetism in fully convective stars.

Motivated by the wealth of observational and theoretical puzzles posed by such stars, we turn in this paper to new 3D MHD simulations of the interiors of low-mass M dwarfs. We aim here to provide additional constraints on the nature of convection and differential rotation in these stars, on the possible dynamo action achieved within their interiors, and on the morphology of the magnetic fields generated by such dynamo action. Our work is most analogous to that of DSB06, but we differ from them in several ways - both in the construction of our model and in the results it produces. In $\S 2$ we describe our numerical model and the principal simplifications adopted, highlighting some of the differences between our simulations and those of DSB06. Section 3 contains a description of the morphology of the convective flows, and an analysis of the spatial variations present in those flows. In $\S 4$ we assess the overall energetics of the dynamo action that is realized, and in $\S 5$ we describe the morphology, strength, and temporal variability of the resulting magnetism. The establishment of differential rotation in hydrodynamic cases, and its quenching in MHD ones, is analyzed in $\S 6$. We assess some aspects of the dynamo process itself in $\S 7$; we close in $\S 8$ with a comparison to prior modeling and observation, and a reflection on what remains to be done.

\section{FORMULATING THE PROBLEM}

\subsection{Fully Convective Rotating Sphere}

The simulations here are intended to be highly simplified descriptions of the interiors of fully convective 0.3 solar-mass M dwarfs. We utilize the Anelastic Spherical Harmonic (ASH) code, which solves within the anelastic approximation the 3D equations that govern fluid motion and magnetic field evolution. Our computational domain is spherical, and extends from $0.08 R$ to $0.96 R$ in radius, with $R$ the overall stellar radius of $2.068 \times 10^{10} \mathrm{~cm}$. We are forced to exclude the inner $8 \%$ of the star from our computations, both because the coordinate systems employed in ASH are singular there, and because the small numerical mesh sizes very near the center of the star would necessitate impractically small time steps. This excluded central region might in principle cause some spurious physical behavior, for instance by projecting a Taylor column aligned with the rotation axis (e.g., Pedlosky 1987) into the surrounding fluid, by giving rise to a central viscous boundary layer, or simply by excluding motions that would otherwise pass through the stellar center. In trial simulations with smaller and larger excluded central regions (ranging from $0.04 R$ to $0.12 R$ ), the properties of the mean flows were very similar to those described here, giving us some confidence that the large-scale dynamics are relatively insensitive to this inner boundary layer. Our computations also do not extend all the way to the stellar surface, because the very low densities in the outer few percent of the star favor the driving of fast, small-scale motions that we cannot resolve.

The initial stratifications of mean density $\bar{\rho}$, energy generation rate $\epsilon$, gravity $g$, radiative diffusivity $\kappa_{\text {rad }}$, and entropy gradient $d S / d r$ are adopted from a $1 \mathrm{D}$ stellar model (I. Baraffe, private communication; cf Chabrier et al. 2000). The initial profiles of the remaining thermodynamic quantities are then given by solving the system of equations described in $\S 2.2$, as described more thoroughly elsewhere (Miesch et al. 2000; BMT04). The thermodynamic quantities are updated throughout the course of the simulation, as the evolving convection modifies the spherically symmetric mean state.

The main parameters of our hydrodynamic and magnetohydrodynamic (MHD) simulations are described in Table 1 . The three hydrodynamic cases $\mathrm{A}, \mathrm{B}$, and $\mathrm{C}$ sample flows of varying complexity, achieved by modifying the effective eddy viscosities and diffusivities $\nu$ and $\kappa$, with case $\mathrm{C}$ the most turbulent (and highest resolution) simulation. Two MHD calculations $\mathrm{Cm}$ and $\mathrm{Bm}$ were begun by introducing small-amplitude seed magnetic fields into the statistically mature progenitor cases $\mathrm{C}$ and $\mathrm{B}$, respectively, and allowing the fields to evolve. A third MHD case $\mathrm{Cm} 2$ was begun from a statistically mature instant in the evolution of case $\mathrm{Cm}$. The two cases $\mathrm{Cm}$ and $\mathrm{Cm} 2$ differ in the magnetic Prandtl number $\mathrm{Pm}=\nu / \eta$ used, which in turn affects the magnetic Reynolds numbers $\mathrm{Rm} \sim u L / \eta$ achieved by the evolved simulations. All the simulations presented here rotate at the solar angular velocity of $\hat{\Omega}=2.6 \times 10^{-6} \mathrm{~s}^{-1}$. In this paper we have chosen for clarity's sake to concentrate our discussion on the two cases $\mathrm{C}$ and $\mathrm{Cm}$. These represent our highest $\mathrm{Re} \sim$ $u L / \nu$ and Rm cases, respectively, so we believe they are likely to be most indicative of the highly turbulent conditions achieved in real stars. Where appropriate, we give some indications of the behavior of the other cases.

\subsection{Anelastic MHD Equations}

ASH solves the 3D MHD anelastic equations of motion in a rotating spherical geometry using a pseudospectral semi-implicit approach (e.g., Clune et al. 1999; Miesch et al. 2000; BMT04). The equations are fully nonlinear in the velocities and magnetic fields, but linearized in thermodynamic variables with respect to a spherically symmetric mean state that is also allowed to evolve. This mean state is taken to have density $\bar{\rho}$, pressure $\bar{P}$, temperature $\bar{T}$, specific entropy $\bar{S}$; perturbations are denoted as $\rho, P, T$, and $S$. The equations solved are

$$
\begin{gathered}
\nabla \cdot(\bar{\rho} \boldsymbol{v})=0 \\
\nabla \cdot \boldsymbol{B}=0 \\
\rho\left[\frac{\partial \boldsymbol{v}}{\partial t}+(\boldsymbol{v} \cdot \nabla) \boldsymbol{v}+2 \boldsymbol{\Omega}_{\boldsymbol{o}} \times \boldsymbol{v}\right]= \\
-\nabla P+\rho \boldsymbol{g}+\frac{1}{4 \pi}(\nabla \times \boldsymbol{B}) \boldsymbol{B} \\
-\nabla \cdot \boldsymbol{D}-[\nabla \bar{P}-\bar{\rho} \boldsymbol{g}],
\end{gathered}
$$


TABLE 1

Simulation Attributes

\begin{tabular}{|c|c|c|c|c|c|c|}
\hline Case & A & B & $\mathrm{C}$ & $\mathrm{Bm}$ & $\mathrm{Cm}$ & $\mathrm{Cm} 2$ \\
\hline \multicolumn{7}{|c|}{ Input Parameters } \\
\hline$\nu\left(\mathrm{cm}^{2} \mathrm{~s}^{-1}\right) \ldots \ldots \ldots \ldots \ldots$ & $5.0 \times 10^{11}$ & $2.2 \times 10^{11}$ & $1.0 \times 10^{11}$ & $2.2 \times 10^{11}$ & $1.0 \times 10^{11}$ & $1.0 \times 10^{11}$ \\
\hline$\kappa\left(\mathrm{cm}^{2} \mathrm{~s}^{-1}\right) \ldots \ldots \ldots \ldots \ldots \ldots \ldots$ & $2.0 \times 10^{12}$ & $8.8 \times 10^{11}$ & $4.0 \times 10^{11}$ & $8.8 \times 10^{11}$ & $4.0 \times 10^{11}$ & $4.0 \times 10^{11}$ \\
\hline 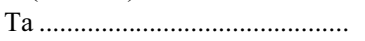 & $1.1 \times 10^{7}$ & $5.9 \times 10^{7}$ & $2.8 \times 10^{8}$ & $5.9 \times 10^{7}$ & $2.8 \times 10^{8}$ & $2.8 \times 10^{8}$ \\
\hline 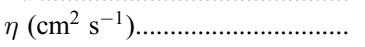 & $\ldots$ & $\ldots$ & $\ldots$ & $2.75 \times 10^{10}$ & $1.25 \times 10^{10}$ & $2.0 \times 10^{10}$ \\
\hline Pm & $\cdots$ & $\cdots$ & $\ldots$ & 8 & 8 & 5 \\
\hline \multicolumn{7}{|c|}{ Measured Quantities } \\
\hline 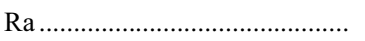 & $3.4 \times 10^{5}$ & $1.4 \times 10^{6}$ & $6.5 \times 10^{6}$ & $1.7 \times 10^{6}$ & $6.3 \times 10^{6}$ & $6.0 \times 10^{6}$ \\
\hline $\operatorname{Re}$ & 65 & 120 & 270 & 110 & 210 & 230 \\
\hline $\mathrm{Rm}$ & $\ldots$ & $\ldots$ & $\ldots$ & 880 & 1650 & 1160 \\
\hline Ro & $1.6 \times 10^{-2}$ & $1.3 \times 10^{-2}$ & $1.3 \times 10^{-2}$ & $1.2 \times 10^{-2}$ & $1.0 \times 10^{-2}$ & $1.1 \times 10^{-2}$ \\
\hline Rc & 0.38 & 0.34 & 0.33 & 0.37 & 0.33 & 0.32 \\
\hline
\end{tabular}

Notes.-The Prandtl number $\operatorname{Pr}=\nu / \kappa=0.25$ for all simulations; the magnetic Prandtl number $\operatorname{Pm}=\nu / \eta$ is indicated for each case, along with the viscosity $\nu$, eddy thermal diffusivity $\kappa$, and magnetic diffusivity $\eta\left(\right.$ in $\mathrm{cm}^{2} \mathrm{~s}^{-1}$ ). The rotation rate $\Omega=2.6 \times 10^{-6} \mathrm{~s}^{-1}$ for all cases. Evaluated as volume averages are the Rayleigh number $\mathrm{Ra}=(-\partial \bar{\rho} / \partial S) \Delta S g L^{3} / \rho \nu \kappa$ (with $\Delta S$ the entropy contrast across the interior), the Taylor number $\mathrm{Ta}=4 \Omega^{2} L^{4} / \nu^{2}$, and the convective Rossby number $\operatorname{Roc}=(\operatorname{Ra} / \mathrm{Ta} \operatorname{Pr})^{1 / 2}$. The Reynolds number $\operatorname{Re}=\tilde{v}^{\prime} L / \nu$, the magnetic Reynolds number $\operatorname{Rm}=\tilde{v}^{\prime} L / \eta$, and the Rossby number Ro $=\tilde{v}^{\prime} / 2 \Omega L$ are evaluated at $r=0.88 R$ using the rms velocity $\tilde{v}$ there. Values based on the maximum velocity would be about a factor of four higher; likewise, values would be slightly higher if based on $\tilde{v}$ closer to the surface, and lower if based on $\tilde{v}$ deeper within the star.

$$
\begin{aligned}
\bar{\rho} \bar{T} \frac{\partial S}{\partial t}+\bar{\rho} \bar{T} \boldsymbol{v} \cdot \nabla(\bar{S}+S) & = \\
& \nabla \cdot\left[\kappa_{r} \bar{\rho} c_{p} \nabla(\bar{T}+T)+\kappa \bar{\rho} \bar{T} \nabla(\bar{S}+S)\right] \\
& +\frac{4 \pi \eta}{c^{2}} \boldsymbol{j}^{2}+2 \bar{\rho} \nu \\
& \times\left[e_{i j} e_{i j}-1 / 3(\nabla \cdot \boldsymbol{v})^{2}\right]+\bar{\rho} \epsilon
\end{aligned}
$$

where $\boldsymbol{g}$ is acceleration due to gravity, $\boldsymbol{v}=\left(v_{r}, v_{\theta}, v_{\phi}\right)$ is the velocity in spherical coordinates in the frame rotating at constant angular velocity $\boldsymbol{\Omega}_{\boldsymbol{o}}, \boldsymbol{B}=\left(B_{r}, B_{\theta}, B_{\phi}\right)$ is the magnetic field, $\boldsymbol{j}=$ $c / 4 \pi(\nabla \times B)$ is the current density, $\kappa_{r}$ is the radiative diffusivity, $c_{p}$ is the specific heat at constant pressure, $\nu$ is the effective eddy viscosity, $\kappa$ is the effective thermal diffusivity, $\eta$ is the effective magnetic diffusivity, and $\mathcal{D}$ is the viscous stress tensor, defined by

$$
\mathcal{D}_{i j}=-2 \bar{\rho} \nu\left[e_{i j}-1 / 3(\nabla \cdot v) \delta_{i j}\right],
$$

with $e_{i j}$ the strain rate tensor. The volume heating term $\bar{\rho} \epsilon$ is included to represent energy generation by nuclear burning of the $\mathrm{CNO}$ cycle within the convective core, and is assumed for simplicity to scale with temperature alone. Our adoption of a subgrid-scale (SGS) heat transport term proportional to the entropy gradient in equation (4) is most justifiable in convection zones, where the stratification will tend toward adiabaticity; in stable zones, this term should be modified in order to avoid spuriously large SGS heat fluxes directed radially inwards (as discussed in Miesch 1998; Miesch et al. 2000). In ASH, we choose to deal with this difficulty by specifying the spherically symmetric $(\ell=0)$ eddy thermal diffusivity $\kappa_{0}$ separately from the $\ell \neq 0$ component $\kappa$; the latter is here taken to be constant in radius, whereas the former increases in a narrow layer near the surface, where the SGS transport must account for the entire outward energy flux (see $\S 3.2$ ). The eddy diffusivity $\kappa$ is in effect purely dissipative, and acts to smooth out entropy variations, whereas $\kappa_{0}$ is essentially a cooling term near the surface. In simulations with stable layers, $\kappa_{0}$ can be modified to account for the presence of radiative regions (e.g., Miesch et al. 2000); here, however, this subgrid transport is small throughout the interior because of the near-adiabatic stratification. To close the set of equations, we take the thermodynamic fluctuations to satisfy the linearized relations

$$
\frac{\rho}{\bar{\rho}}=\frac{P}{\bar{P}}-\frac{T}{\bar{T}}=\frac{P}{\gamma \bar{P}}-\frac{S}{c_{p}},
$$

assuming the ideal gas law

$$
\bar{P}=\mathcal{R} \bar{\rho} \bar{T},
$$

where $\mathcal{R}$ is the gas constant. The effects of compressibility are included via the anelastic approximation, which filters out sound waves that would otherwise limit the time steps allowed by the simulation to the sound crossing time across the smallest computational zone. In the low-Mach-number flows typical of stellar interior convection, adopting the anelastic approximation allows us to take much larger time steps that satisfy the Courant-FreidrichsLewy condition imposed by the convective flows, rather than the much shorter one required to follow acoustic waves. In the MHD simulations, the anelastic approximation filters out fast magnetoacoustic modes but retains the Alfvén and slow magnetoacoustic modes. We use a toroidal-poloidal decomposition for the mass flux and magnetic field in order to ensure that both remain divergencefree throughout the simulation, with

$$
\begin{aligned}
\bar{\rho} \boldsymbol{v} & =\nabla \times \nabla \times\left(W \boldsymbol{e}_{r}\right)+\nabla \times\left(Z \boldsymbol{e}_{r}\right), \\
\boldsymbol{B} & =\nabla \times \nabla \times\left(C \boldsymbol{e}_{r}\right)+\nabla \times\left(A \boldsymbol{e}_{r}\right),
\end{aligned}
$$

with $\boldsymbol{e}$ a unit vector, and involving the streamfunctions $W$ and $Z$ and magnetic potentials $C$ and $A$.

This system of equations requires 12 boundary conditions and suitable initial conditions. Because one of our aims is to assess the angular momentum redistribution in our simulations, we have opted for torque-free velocity boundary conditions at the top and 
bottom of the deep spherical domain. We have assumed the following:

1. Impenetrable top and bottom surfaces: $v_{r}=\left.0\right|_{r=r_{\text {bot }}, r_{\text {top }}}$.

2. Stress-free top and bottom: $\partial / \partial r\left(v_{\theta} / r\right)=\partial / \partial r\left(v_{\theta} / r\right)=$ $0 \mid r=r_{\text {bot }}, r_{\text {top }}$.

3. Constant entropy gradient at top and bottom: $\partial \bar{S} / \partial r=$ constant $\left.\right|_{r=r_{\text {bot }}, r_{\text {top }}}$.

4. Match to an external potential field at the top: $\boldsymbol{B}=\nabla \Phi \rightarrow$ $\Delta \Phi=\left.0\right|_{r=r_{\text {top }}}$, and to a perfect conductor (purely tangential field) at the bottom.

In the analysis that follows, we often form various spatial and temporal averages of the evolving convective flows and magnetic fields. For clarity, we note that we use the symbol $\hat{a}$ to indicate temporal and longitudinal averaging of a variable $a$, and the symbol $\langle a\rangle$ to denote longitudinal averaging to obtain the axisymmetric component of the variable. Such averaging allows us to separate the fluctuating (denoted by a prime as $a^{\prime}$ ) from the axisymmetric (mean) parts of the variable. The symbol $\tilde{a}$ designates the rms average of $a$, carried out on a spherical surface for many realizations in time. Likewise, the combined symbols $\tilde{a}^{\prime}$ represent rms averaging of the variable from which the axisymmetric portion has been subtracted.

\subsection{Numerical Approach}

No numerical simulation can model with perfect fidelity the intensely turbulent convection occurring in stars. The range of spatial scales present in such convection is too vast; some simplification is unavoidable. We choose in our global modeling to resolve the largest scales of motion, which we believe are likely to play dominant roles in redistributing energy and angular momentum and in building magnetic fields. Our simulations are therefore classified as large-eddy simulations (LES), with the effects of unresolved small scales of turbulence incorporated using a subgrid-scale (SGS) treatment. Here these unresolved scales are treated simply as enhanced viscosities and thermal and magnetic diffusivities ( $\nu, \kappa$, and $\eta$ respectively), which are thus effective eddy viscosities and diffusivities. For simplicity, we have taken these to be constant in radius. This implies that the viscous damping at depth in our simulations may be too severe relative to that near the surface. More sophisticated SGS treatments, in which $\nu$ and $\kappa$ are proportional to properties of the resolved flow field (e.g., the velocity or strain rate) would certainly be desirable, and we hope to explore such strategies in future work. Our simulations are characterized by nondimensional numbers relating these diffusivities to one another and to the various terms in the momentum equation (3); some of these dimensionless numbers necessarily take on very different values in our modeling than in actual stellar interiors. We have adopted a magnetic Prandtl number Pm $=\nu / \eta>1$ in our MHD cases, even though a Pm based on microscopic viscosities and diffusivities would be much less than unity. The large Pm here reflects unresolved turbulent mixing processes, and allows us to achieve moderately high values of the magnetic Reynolds number $\mathrm{Rm}=$ $u L / \eta$ with tractable numerical resolution. At small Pm, the critical Rm needed for dynamo action increases considerably (Boldyrev \& Cattaneo 2004; Schekochihin et al. 2005), rendering simulations more computationally demanding. The strength and morphology of the magnetic fields realized in our simulations are likely sensitive at some level to the Pm and Rm chosen. We are somewhat encouraged by prior simulations of convection in the solar interior in comparable parameter regimes (Brun \& Toomre 2002; Brun et al. 2005, hereafter BBT05; Browning et al. 2006), for these have been relatively successful in matching the detailed observational constraints on, e.g., solar differential rotation provided by helioseismology.

The numerical strategies employed within ASH are described in detail elsewhere (Clune et al. 1999; BMT04); we here summarize only a few key features. The dynamical variables within ASH are expanded in terms of spherical harmonic basis functions $Y_{l}^{m}(\theta, \phi)$ in the horizontal directions and Chebyshev polynomials $T_{n}(r)$ in the radial. The spatial resolution is thus kept uniform everywhere on a spherical surface by employing a complete set of spherical harmonics of degree $\ell$, retaining all azimuthal orders $m$ in what is referred to as a triangular truncation. We limit our expansion to a degree $\ell=\ell_{\max }$, related to the number of latitudinal mesh points $N_{\theta}$ by $\ell_{\max }=\left(2 N_{\theta}-1\right) / 3$, take $N_{\phi}=2 N_{\theta}$ longitudinal mesh points, and use $N_{r}$ colocation points for the radial projection onto Chebyshev polynomials. Our highest resolution simulations here $(\mathrm{Cm})$ have $\ell_{\max }=340$ (implying $N_{\theta}=512$ and $\left.N_{\phi}=1024\right)$ and $N_{r}=192$. We employ an implicit, second-order Crank-Nicholson scheme for calculating the time evolution of the linear terms in equations (1)-(5), whereas an explicit second-order Adams-Bashforth scheme is used for the advective, Lorentz, and Coriolis terms. ASH runs efficiently on a variety of massively parallel supercomputers using the message passing interface (MPI). The code's performance scales reasonably well up to about 1000 processors. The simulations described here required roughly half a million hours of computing time.

Our computational approach differs from that of DSB06 in a few important ways. Because theirs is the only prior 3D MHD simulation of fully convective stars, we here comment briefly on these differences. In order to keep thermal relaxation timescales small, the simulations in DSB06 adopted a stellar luminosity roughly $10^{12}$ times higher than appropriate for an actual $\mathrm{M}$ dwarf. This choice is related to the fact that DSB06 adopt the same thermal diffusivities for the mean temperature gradient and for the small-scale turbulent temperature fluctuations; in our modeling, the mean temperature gradient is acted on by the thermal diffusivity $\kappa_{r}$ taken from a 1D stellar model, whereas $\kappa$ for the turbulent temperature field is (as in DSB06) a subgrid-scale eddy diffusivity. Our strategy allows us to assess with reasonable fidelity the radiative flux within the interior, since $\kappa_{r}$ in our models is ultimately set by the radiative opacities of the 1D stellar model. The DSB06 rescaling of the luminosity implies a commensurate artificial increase in the typical convective velocities. In order to keep the ratio between the convective turnover times and the rotation period approximately correct, they also considered very rapid rotation rates. In terms of the nondimensional numbers Re, Rm, and Ro, our simulations are roughly comparable to theirs. However, it is difficult to gauge with certainty the impact that their rescalings of $L, v_{c}$, and $\Omega$ may have on the resulting flows and magnetic fields. We have chosen not to perform such a rescaling, which means that very long term adjustments of the mean temperature gradient would not be captured in our modeling. Both strategies for dealing with the thermal diffusivity have been widely employed; neither is perfect. Our simulations also differ from those of DSB06 in a few smaller ways. The overall density contrast between the inner and outer boundaries in our models is about 100 , consistent with the contrast between $0.1 R$ and $0.96 R$ in the $1 \mathrm{D}$ stellar model we used for our initial conditions. In DSB06, the density varied by a factor of about 5 from center to surface; the larger density contrasts in our modeling have a substantial impact on the morphology of the convective flows. The boundary conditions adopted in DSB06 also differ from ours; theirs is closer to a no-slip boundary condition than to the stress-free boundaries used here. This difference may impact our results on the differential rotation realized in hydrodynamic 

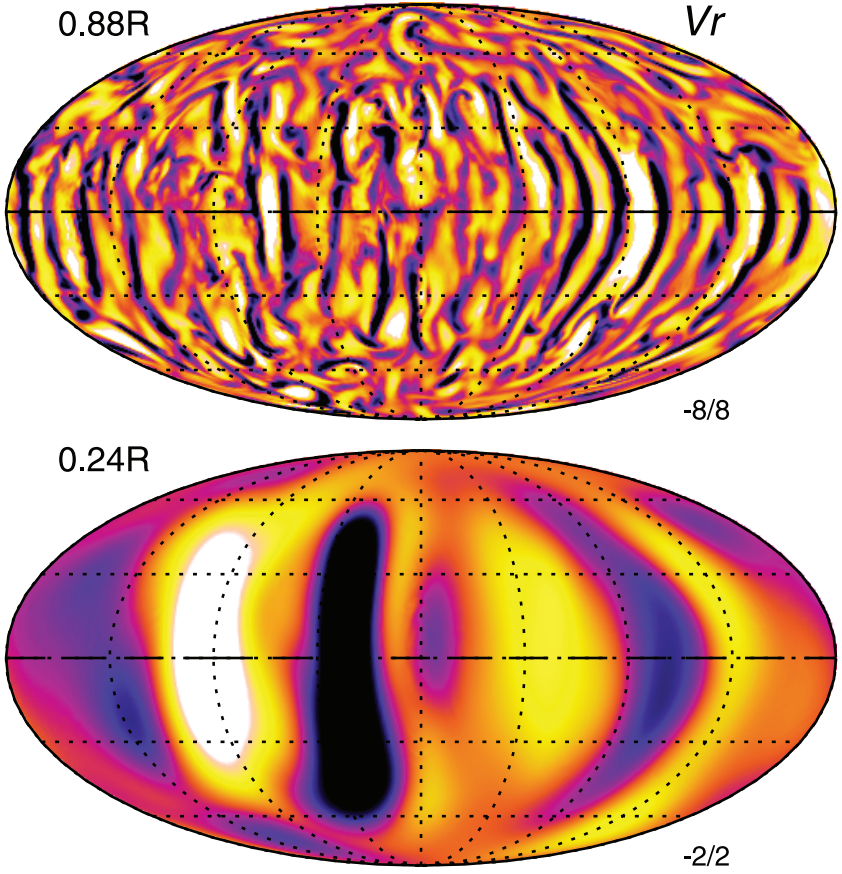

FIG. 1.-Radial velocity $v_{r}$ on spherical surfaces at two depths for a single instant in the evolution of case $\mathrm{Cm}$. Upflows are rendered in red tones, and downflows in blue; the maxima and minima of the colormaps are indicated. The flows are stronger and on smaller spatial scales near the surface than they are are depth.

simulations ( $\S 6$ ). Because they employed a Cartesian finitedifference code, DSB06 were able to model the central few percent of the star, omitted in our modeling. These factors may all contribute to differences between the results here and in DSB06. Nonetheless, there is some common ground between our findings and theirs; we comment more on both the similarities and differences of the two models in $\S 8$ below.

\section{CONVECTIVE FLOWS AND ENERGY TRANSPORT}

\subsection{Morphology of the Flows}

The convective flows realized in our simulations possess structure on many spatial scales. An instantaneous view of the flows in the hydrodynamic case $\mathrm{C}$ is provided by Figure 1, which shows the radial velocity $v_{r}$ near both the top of the computational domain (Fig. $1 a$, at $r=0.88 R$ ) and deeper within the interior (Fig. $1 b$, at $r=0.24 R$ ). At large radii, a marked asymmetry between upflows and downflows is apparent (Fig. 1a), with the downflows compact and strong while upflows are broader and weaker. This asymmetry is driven mainly by the strong density stratification at these radii: downflows tend to contract, whereas upflows expand. Some of these downflow lanes persist as coherent structures for extended intervals, while many complex and intermittent features also appear on smaller scales. Such coherent downflow plumes have previously been noted as a seemingly generic feature of turbulent compressible convection (e.g., Brummell et al. 2002; Brun \& Toomre 2002).

In Figure $1 a$ these downflows generally appear aligned with the rotation axis at low latitudes; at high latitudes the distribution of upflows and downflows is more isotropic. Similar distinctions between flows at high latitudes versus those near the equator have often been realized in simulations of the solar convective envelope (e.g., Miesch et al. 2000), where the difference was identified with the "tangent cylinder" formed by projecting the lower boundary at the equator onto the upper boundary.
In those simulations, the inner boundary prohibited connectivity between the northern and southern hemispheres for flows far from the equator, whereas motions at low latitudes could readily couple both hemispheres. Here, the inner boundary is sufficiently deep to allow connectivity between high-latitude flows as well, but motions that span both hemispheres are still realized only near the equator. This may arise because the effects of the Coriolis forces, which ultimately drive turbulent alignment with the rotation axis, still vary with distance from the rotation axis (see, e.g., Busse 1970); the low-latitude flows may also reflect a lingering preference for the most unstable modes in a rotating spherical shell, which at these rotation rates tend to be symmetric fluid rolls perpendicular to the equator (e.g., Gilman 1976).

Deeper within the interior (Fig. 1b), the convection is characterized by broader, weaker flows that span large fractions of a hemisphere. Upflows and downflows are fairly symmetric in appearance there, likely because the density stratification at depth is weaker. The density scale height $\lambda_{p}=P / g \rho$ varies from about $10^{9} \mathrm{~cm}$ at $r=0.88 R$ to $4 \times 10^{9} \mathrm{~cm}$ at $r=0.24 R$, which corresponds roughly to the physical size of the convective cells at the two radii; note that the spatial size of the convective patterns, not just their angular size, varies with radius. The convective patterns at $r=0.24 R$ are reminiscent of sectoral spherical harmonics $Y_{m}^{\ell}$ with $\ell=m=3$; at other instants in the evolution of case $\mathrm{C}$, this identification is stronger. The motions deep in the interior are linked to those farther out: small downflow plumes at large radii merge as they descend, and coalesce to form the broad downflows seen in Figure $1 b$. This coupling between depths is weakly discernible in Figure 1: the most striking downflow plumes at $r=0.88 R$ generally occur in regions where downflow persists at $r=0.24 R$.

The amplitude of the convective motions also changes with depth. Typical rms velocities $\tilde{v}$ at $r=0.88 R$ are about $12 \mathrm{~m} \mathrm{~s}^{-1}$, whereas at $r=0.24 R, \tilde{v} \approx 2 \mathrm{~m} \mathrm{~s}^{-1}$. This variation, together with the smaller typical eddy sizes $l_{\text {eddy }}$ near the surface, means that the local convective overturning time $\tau_{c} \sim l_{\text {eddy }} / \tilde{v}$ varies by a factor of about 20 across the domain. For small-scale local dynamo action, the characteristic timescale for field amplification is roughly the convective turnover time (e.g., Childress \& Gilbert 1995); thus, we might expect that fields will be built more rapidly in the outer layers of the star than at depth. We see in $\S 5$ that this is indeed the case.

Thus, although the interior is unstably stratified at all depths, there are still two conceptually distinct regions: one near the surface in which convection is vigorous, possesses a variety of small-scale structure, and might quickly amplify any seed magnetic fields; and another at depth where the flows are more quiescent, with large-scale overturning motions that may amplify fields somewhat more slowly. In the following subsection, we examine why the vigor of convection may vary with depth.

\subsection{Spatial Variation of Energy Transport}

Convection in stars is driven ultimately by the need to transport energy outwards. That energy arises primarily from nuclearburning reactions within the central regions of the star, so the total luminosity $L(r)$ that must be carried outwards is an increasing function of radius, out to the point where nuclear burning stops and $L(r)$ is equal to the surface luminosity $L_{*}$. In Figure 2 we assess for simulation $\mathrm{C}$ the radial transport of energy by different physical processes, defined as

$$
F_{c}+F_{k}+F_{\mathrm{r}}+F_{u}+F_{v}=\frac{L(r)}{4 \pi r^{2}},
$$




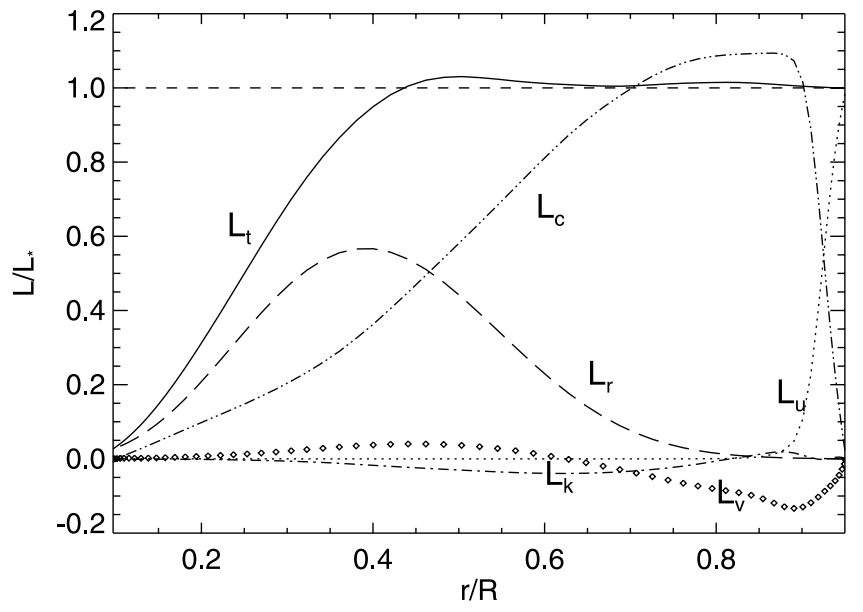

FIG. 2.- Time-averaged radial transport of energy in case C. Shown are the convective enthalpy flux $F_{c}$, radiative flux $F_{r}$, viscous flux $F_{v}$, kinetic energy flux $F_{k}$, the unresolved flux $F_{u}$, and their sum the total flux $F_{t}$; all have been expressed as luminosities. Although the stratification is convectively unstable everywhere, the convective flux carries most of the energy only at radii larger than about $0.45 R$.

with

$$
\begin{gathered}
F_{c}=\bar{\rho} c_{p} \overline{v_{r} T^{\prime}}, \\
F_{k}=\frac{1}{2} \bar{\rho} \overline{v^{2} v_{r}}, \\
F_{r}=-\kappa_{r} \bar{\rho} c_{p} \frac{d \bar{T}}{d r}, \\
F_{u}=-\kappa \bar{\rho} \bar{T} \frac{d \bar{S}}{d r}, \\
F_{v}=-\overline{\boldsymbol{v} \cdot \mathcal{D}},
\end{gathered}
$$

where the overbar denotes an average over spherical surfaces and in time, $F_{c}$ is the enthalpy flux due to resolved convective flows, $F_{k}$ the kinetic energy flux, $F_{r}$ the radiative flux, $F_{u}$ the unresolved eddy flux, and $F_{v}$ the viscous flux. The unresolved eddy flux $F_{u}$ is the enthalpy flux from subgrid-scale motions that we cannot resolve, which in ASH takes the form of a thermal diffusion operating on the mean entropy gradient. In MHD simulations $(\mathrm{Cm}, \mathrm{Bm}$, and $\mathrm{Cm} 2)$, the Poynting flux $F_{m}=(c / 4 \pi) \overline{E_{\theta} B_{\phi}-E_{\phi} B_{\theta}}$ also contributes, but is small. The viscous flux and the kinetic energy flux are generally small compared to $F_{c}(r)$ and $F_{r}(r)$; the unresolved flux becomes large near the surface, where it carries all the energy because the radial velocity is forced to vanish at the upper boundary.

Figure 2 shows that the radiative flux carries most of the energy at small radii, with $L_{r} \approx 0.70 L(r) \approx 0.10 L_{*}$ at $r=0.15 R$. The enthalpy flux is smaller there, with $L_{c} \approx 0.30 L_{r}=0.04 L_{*}$. Moving to larger radii, however, the total luminosity $L(r)$ rises up to $L_{*}$ in accord with the continuing nuclear energy generation, while at the same time the radiative luminosity $L_{r}(r)$ decreases. Thus, the energy that must be transported by convection goes up significantly, rising to a maximum of $L_{c} \approx 1.1 L_{*}$ at $r=0.80 R$; the excess over the stellar luminosity is mostly compensated by an inward-directed viscous flux. Although convection carries as little as $30 \%$ of the local luminosity near the stellar center, the overall entropy stratification is still nearly adiabatic. The radiative flux is fixed by the radiative opacity, here input from the 1D stellar model, and by the mean temperature gradient; likewise, the variation in the total luminosity with radius is set by the nuclear energy generation rate $\epsilon(r)$, also input from the 1D model.
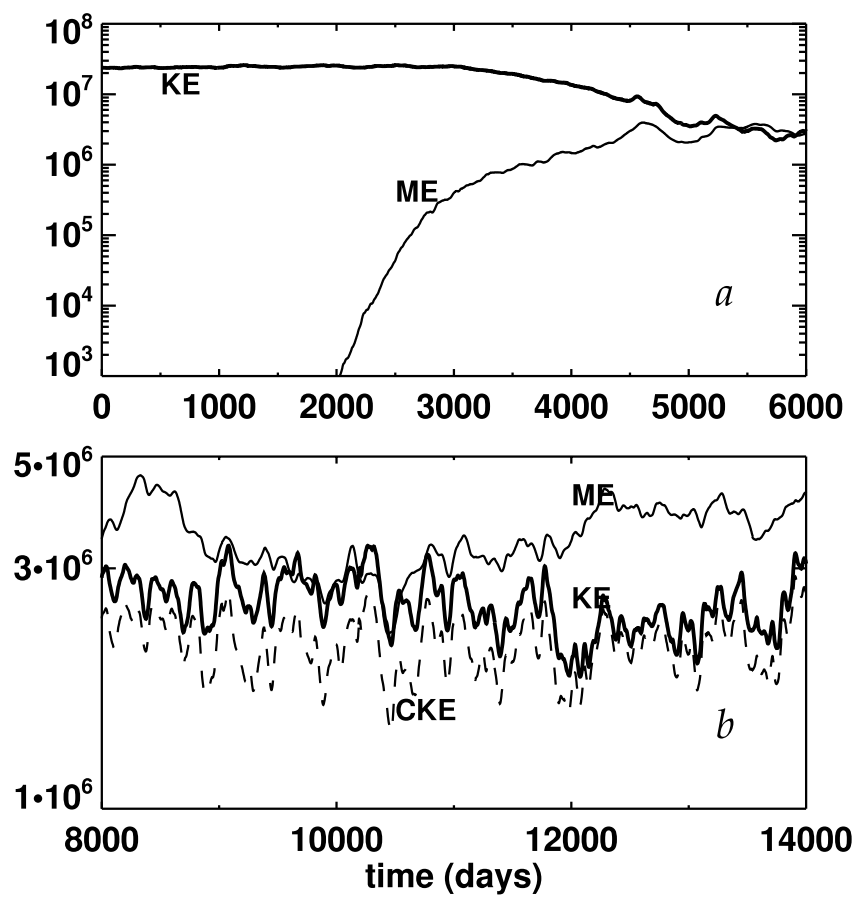

FIG. 3.- Temporal evolution of the volume-averaged magnetic and kinetic energy densities in case $\mathrm{Cm}$. (a) The magnetic energy density (ME) grows by many orders of magnitude from its initial seed value, and ultimately equilibrates when comparable to the kinetic energy density $(\mathrm{KE})$ relative to the rotating frame. (b) Detailed view of the evolution of $\mathrm{KE}$ and $\mathrm{ME}$ during an interval after equilibration was reached; also shown is the energy density in the convection (CKE). On average, the magnetic energy is about $120 \%$ of KE and $140 \%$ of CKE.

To the extent that these input properties are accurate, and assuming there are no drastic long-term changes in the prevailing temperature gradient (which we could not capture in our limited simulation time), we believe that the significant variation of $L_{c}$ with radius is likely to be a robust feature. However, lower mass stars than those considered here might have smaller radiative fluxes in the deep interior, owing to their lower central temperatures and hence higher opacity from metals, and so may exhibit less radial variation of $L_{c}$.

This variation in the energy that must be transported by convection is linked to the radial change in convective velocity noted in $\S 3.1$. On dimensional grounds, the typical convective velocity is given roughly by $v_{c} \propto\left[L_{c}(R / M)\right]^{1 / 3}$, so the factor of 25 change in $L_{c}$ from $r=0.15 R$ to $0.88 R$ would correspond to a factor of about 3 change in typical convective velocity. This assumes that the correlations between temperature fluctuations and the radial velocity field do not change appreciably with depth; in practice, the energy transport is somewhat less efficient amid the highly turbulent flows near the surface, with many regions where temperature and radial velocity are not as well correlated as they are at depth. This effect, plus the strong variation of density with radius, leads to even larger radial variations of $v_{c}$ than this simple scaling argument would suggest.

\section{DYNAMO ACTION REALIZED}

Magnetic dynamo action is achieved by the flows. Small initial seed fields are amplified by several orders of magnitude until they reach a statistically equilibrated state in which their growth is balanced by Ohmic decay. The growth and saturation of the magnetic energy density in case $\mathrm{Cm}$ is displayed in Figure $3 a$, while a phase of evolution after saturation is examined in Figure $3 b$. Also shown there are the kinetic energy densities due to 
nonaxisymmetric motions, which we term the convective kinetic energy density (CKE) and the total kinetic energy density $\mathrm{KE}$ (which is the sum of CKE, the energy in differential rotation DRKE, and the energy in meridional circulations MCKE). All are shown relative to the frame rotating at $\Omega=2.6 \times 10^{-6} \mathrm{~s}^{-1}$. In the evolved simulation, MCKE is approximately 300 times smaller than CKE, and DRKE is likewise a factor of 5 smaller than CKE, so we have omitted them from Figure 3 for clarity.

The magnetic energy in the simulations grows exponentially until it is approximately in equipartition with the flows. Over the last 200 days of simulation $\mathrm{C} 4 \mathrm{~m}$, a period during which no sustained growth or decay of the various energy densities was evident, ME was approximately $120 \%$ of KE and about $140 \%$ of CKE. The initial seed value of ME was about $10^{-2} \mathrm{ergs} \mathrm{cm}^{-3}$, and the phase of exponential growth lasted about 2500 days, implying a characteristic $e$-folding timescale for magnetic energy growth of about 150 days. This may be compared to the typical eddy convective turnover times in the simulation, which vary from about 20 days near the surface to roughly 450 days at depth.

As the magnetic fields grow, they react back on the flows that generated them through the $\boldsymbol{j} \times \boldsymbol{B}$ force term in equation (1). The net effect is to begin to reduce $\mathrm{KE}$ once $\mathrm{ME}$ reaches a threshold value of about $5 \%$ of $\mathrm{KE}$; this is visible in Figure 3 starting at about 3000 days. The reduction in KE is associated mainly with a large decline in DRKE, whereas CKE appears largely unaffected by the growing fields. In the kinematic phase, DRKE is approximately 6 times CKE; after saturation of the dynamo, DRKE/ CKE is only about $0.2-0.4$. Although the energy densities exhibit no systematic variation after the initial phase of dynamo growth, they still show substantial short-term stochastic fluctuations. During the interval sampled by Figure 3, CKE varies by factors of about 3 , and with it the total KE; the magnetic energy density varies by similar amounts. Thus, although $\mathrm{ME} \approx 1.2 \mathrm{KE}$ on average, it can rise as high as twice $\mathrm{KE}$ for short intervals.

The other MHD simulations behave in a similar fashion. In case $\mathrm{Cm} 2$, which has a lower Pm and hence lower Rm (implying a less supercritical dynamo), ME equilibrates at about $90 \%$ of $\mathrm{KE}(120 \%$ of CKE). The weaker magnetic fields in that case lead to a slightly smaller reduction in DRKE than is realized in case $\mathrm{Cm}$. Case $\mathrm{Bm}$, which is both more laminar (lower Re) and less supercritical (lower $\mathrm{Rm}$ ) than the other two simulations, has still lower magnetic energy densities, with $\mathrm{ME} \approx 50 \%$ of KE. These lower magnetic energy densities imply even less quenching of DRKE, which varies between about 0.8 and 1.1 times CKE.

It is instructive to compare these energy densities to those realized in other convective dynamo simulations. In numerical models of the solar convective envelope (with $\mathrm{Pm}=5$ and $\mathrm{Rm} \approx$ 490), BMT04 found that ME equilibrated at about $7 \%$ of KE; Browning et al. (2006) found comparable values within the convective envelope in simulations of the convection zone and a forced tachocline, there adopting $\mathrm{Pm}=8$. In modeling fully convective stars with the PENCIL code, DSB06 found ME comparable to $\mathrm{KE}$ in their most rapidly rotating runs. Models of core convection in A-type stars (BBT05) yielded ME/KE typically between 0.28 and 0.90 , depending on rotation rate and other simulation parameters. In those models, DRKE was strongly suppressed whenever ME was greater than about $30 \%$ of KE, leading to cyclical growth and decay of ME and DRKE: large differential rotation led to growing ME, but the resulting strong fields tended to suppress DRKE, which in turn resulted in a decline in ME. No cyclical behavior of this sort is observed in case $\mathrm{Cm}$; instead, $\mathrm{ME}$ is always much greater than DRKE. The more laminar case $\mathrm{Bm}$, with a lower $\mathrm{ME} / \mathrm{KE}$ ratio, does show some linked oscillations in DRKE and ME, suggesting that such inter- twined feedback is realized only for a fairly narrow range of magnetic energy densities. We believe that case $\mathrm{Cm}$ is likely to be more representative of the behavior of actual stellar interiors at this rotation rate: if anything, the extraordinariliy high $\mathrm{Re}$ and $\mathrm{Rm}$ realized in stars might be expected to lead to somewhat higher magnetic energy densities than we find here, and hence perhaps to an even stronger decline in DRKE.

\section{PROPERTIES OF THE MAGNETIC FIELDS}

\subsection{Morphology and Spatial Distribution}

The magnetic fields realized here possess both intricate smallscale features and global-scale structures. Like that of the flows that sustain them, the morphology of the fields varies with radius, with the typical length scale of the field increasing with depth. A sampling of such behavior is provided by Figure 4, which shows an instantaneous view of the radial magnetic field $B_{r}$, the azimuthal magnetic field $B_{\phi}$, and the radial velocity $v_{r}$ on spherical surfaces at two depths in case $\mathrm{Cm}$. Near the surface, complex structures on many different scales continually emerge and evolve, ranging from localized ripples to large-scale patches in $B_{\phi}$ that extend around much of the domain. The smallest field structures are typically on finer scales than the smallest flow fields, likely partly because we have adopted a magnetic Prandtl number Pm greater than unity. The strongest radial fields of both polarities are generally associated with strong downflow plumes, but only slightly weaker fields may be found in the relatively quiescent regions between these plumes. The field strengths vary somewhat as a function of depth, with typical $B_{r}$ and $B_{\phi}$ sampled by Figure 4 declining by about a factor of 2 in going from $r=0.88 R$ to $0.24 R$.

Deeper within the star (Figs. $4 d-4 f$ ), the magnetic fields are larger in scale. At these depths, the field is no longer structured on appreciably finer scales than the flow, as revealed either by Figures $4 d-4 f$ or by the spectral analysis of $\S 5.2$ below. Like the convective flows, the magnetic fields at depth are coupled to those at larger radii, with the intricate field structures near the surface emerging from the broader network of magnetism below. The fields deep within the interior evolve much more slowly than the small-scale magnetism near the surface, with some large patterns in $B_{\phi}$ (Fig. $4 f$ ) persisting for thousands of days.

In the following subsections, we examine more quantitatively the strength of the fields on both large and small spatial scales.

\subsection{Spatial Scales of the Magnetism}

One assessment of the overall field morphology is provided by decomposing the magnetism into its azimuthal mean (the axisymmetric field) and fluctuations about that mean. This is a coarse measure of the size of typical field structures: if the field is mostly on small scales, only a small signal will survive the azimuthal averaging. We define the shell-averaged toroidal mean magnetic energy (TME), the fluctuating magnetic energy (FME), and the total magnetic energy as follows:

$$
\begin{gathered}
\mathrm{ME}=\frac{1}{8 \pi}\left(B_{r}^{2}+B_{\theta}^{2}+B_{\phi}^{2}\right) \\
\mathrm{TME}=\frac{1}{8 \pi}\left\langle B_{\phi}\right\rangle^{2} \\
\mathrm{FME}=\frac{1}{8 \pi}\left[\left(B_{r}-\left\langle B_{r}\right\rangle\right)^{2}+\left(B_{\theta}-\left\langle B_{\theta}\right\rangle\right)^{2}+\left(B_{\phi}-\left\langle B_{\phi}\right\rangle\right)^{2}\right],
\end{gathered}
$$

recalling that the angle brackets \langle\rangle denote a longitudinal average. These energy components are displayed for case $\mathrm{Cm}$ in Figure 5. 

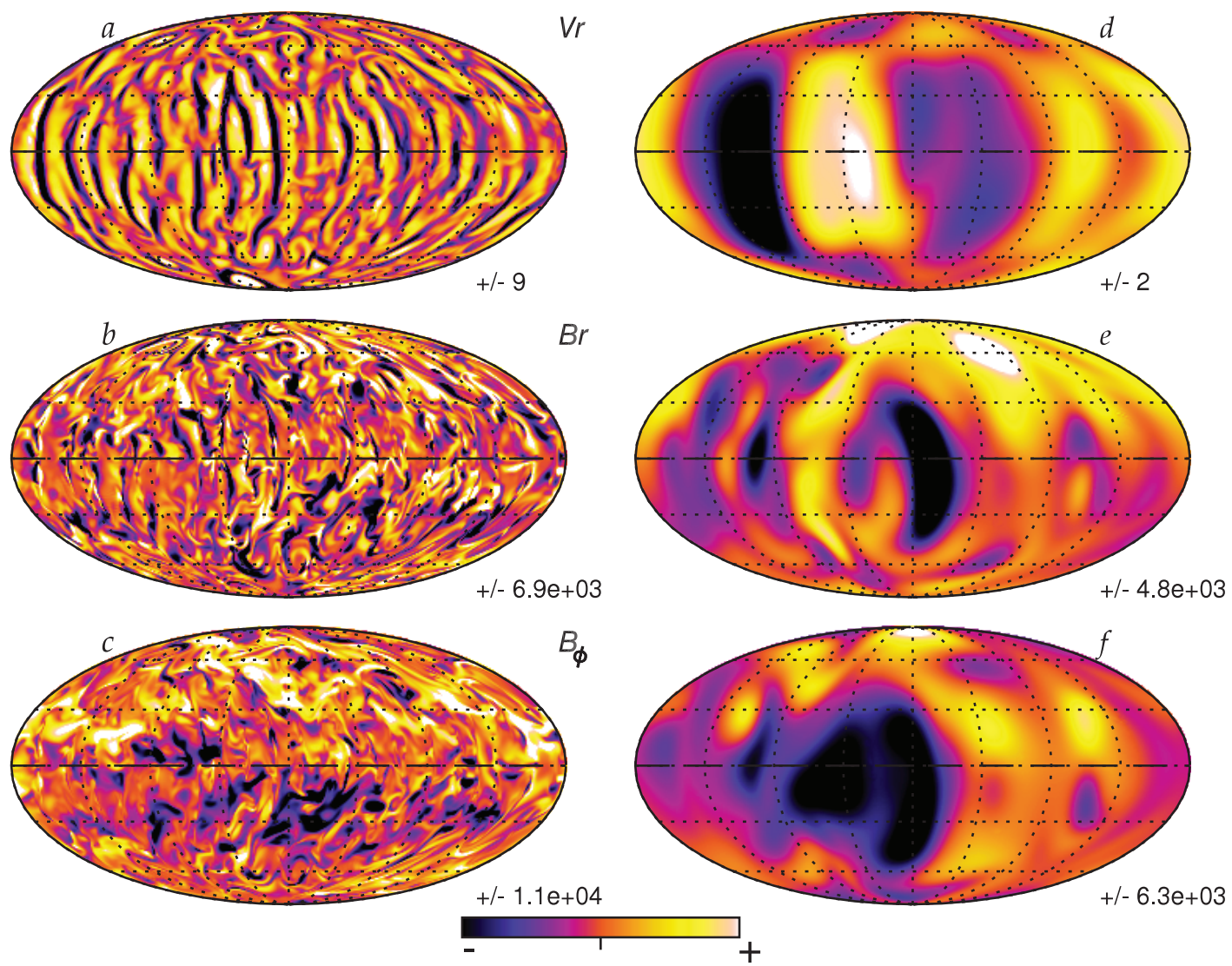

Fig. 4. - Global views of radial velocity $v_{r}$, radial magnetic field $B_{r}$, and longitudinal magnetic field $B_{\phi}$ at a single instant in the evolution of case Cm. All are shown on spherical surfaces both deep within the star (at $r=0.24 R$ ) and closer to the surface $(r=0.88 R$ ), with red tones indicating positive polarity (upflows) and blue tones negative polarity (downflow). Maxima and minima of the color maps are indicated (in $\mathrm{m} \mathrm{s}^{-1}$ and $\mathrm{G}$ ).

Although the majority of the magnetic energy is in the nonaxisymmetric component, the mean toroidal field is still considerable. Within the bulk of the interior, TME accounts for about $18 \%$ of the total magnetic energy; it is smallest near the surface, where $\mathrm{TME} \approx 5 \%$ of $\mathrm{ME}$, and largest (as a fraction of ME) at depth.

That the toroidal mean fields account for a reasonably large fraction of the total ME is a striking result. In prior simulations of the bulk of the solar convective envelope, TME was typically only about $3 \%$ (BMT04); in simulations including a tachocline

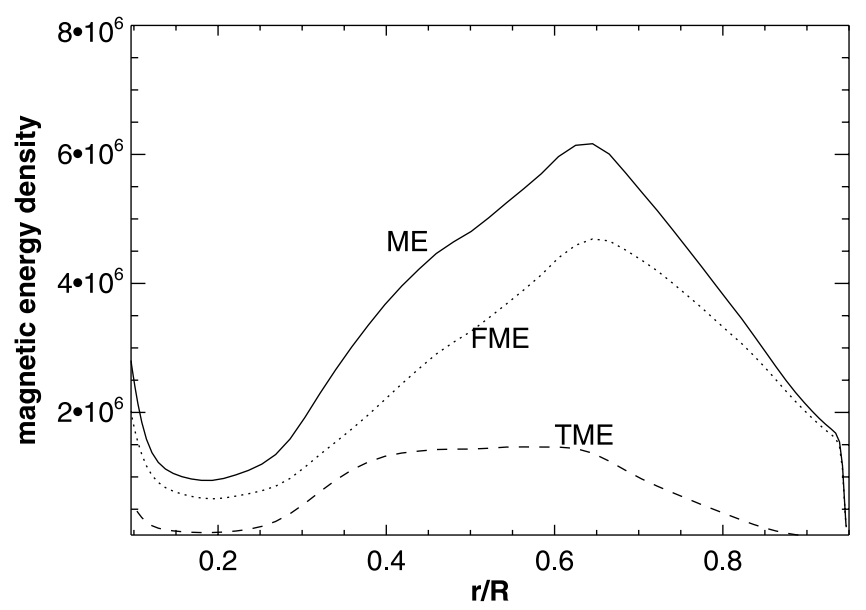

FIG. 5.- Magnetic energy components in case $\mathrm{Cm}$ as a function of radius. Shown are the toroidal mean (axisymmetric) magnetic energy TME, the fluctuating (nonaxisymmetric) magnetic energy FME, and their sum the total magnetic energy ME, all averaged in time and over spherical surfaces. of shear, similar TME/ME ratios to those reported here were attained only within the stably stratified tachocline itself (Browning et al. 2006). Similarly, BBT05 found that $\mathrm{TME} / \mathrm{ME} \approx 0.05$ within most of the convective cores of A-type stars, with higher values achieved only within a shear layer at the boundary of that core.

To glean a more complete understanding of the spatial structure of the magnetic fields and the flows that sustain them, we turn to the spatial power spectra shown in Figure 6. There the time-averaged $B^{2}$ is shown at selected depths as a function of spherical harmonic degree $\ell$ (Fig. $6 a$ ), along with the velocity spectra $v^{2}$ for comparison (Fig. $6 b$ ). The velocity spectra broadly confirm the qualitative descriptions of $\S 3.1$ : at large radii, the spectra peak at higher wavenumbers (smaller spatial scales) than at small radii. Near the surface, the velocity amplitudes rise gradually from low $\ell$ to a peak at about $\ell=20$, with all spherical harmonic degrees $\ell<35$ possessing as much power as the $\ell=1$ mode. The velocity amplitudes fall of steeply with increasing $\ell$ beyond the peak, approximating a power law of slope steeper than $\ell^{-2}$. In the deeper interior, the spectra also show a comparatively gradual rise to a maximum amplitude at a scale $\ell_{\text {peak }}$, and a steep falloff to higher $\ell$, but the value of $\ell_{\text {peak }}$ shifts to smaller $\ell$. At the smallest radii sampled here, the slope of the velocity spectra becomes somewhat shallower around $\ell=20$. One significant caveat is that our choice of eddy viscosities and diffusivities that are constant with depth is somewhat arbitrary; if these coefficients were instead taken to decrease with decreasing radius (in order to crudely represent kinetic energy dissipation by smallscale turbulence that is constant in radius), the contrast between the flows at depth and those near the surface would likely not be as great as that reported here. 

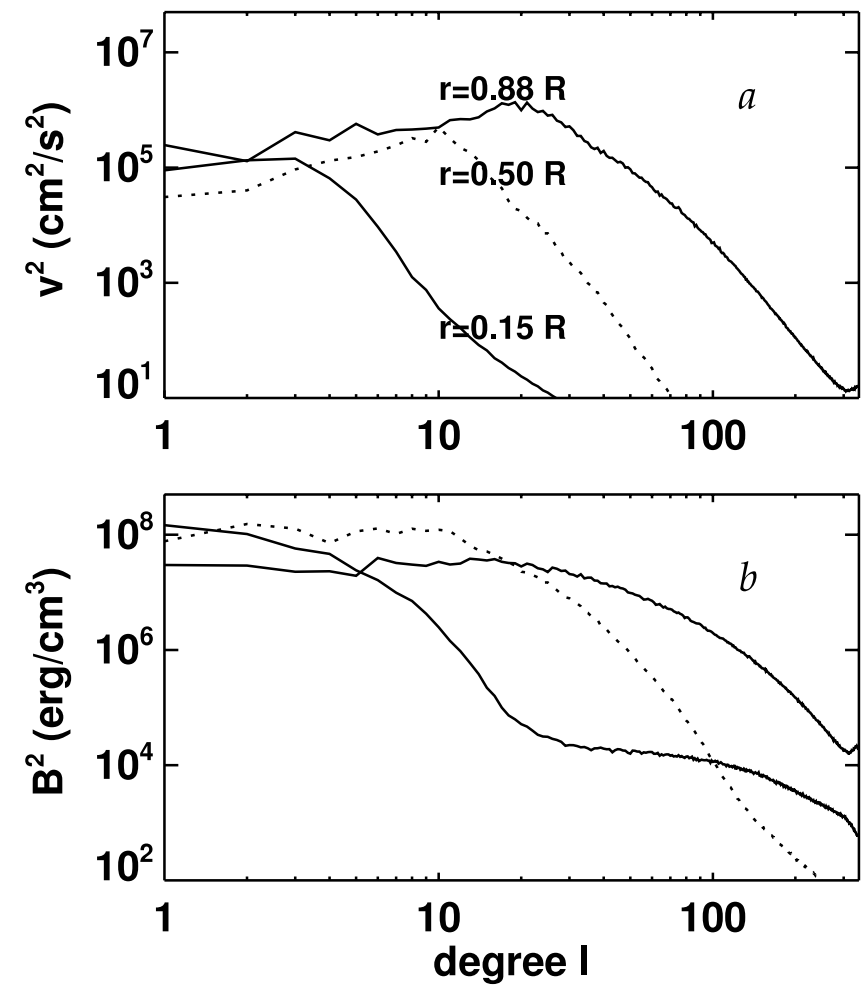

Fig. 6.- Time-averaged spectral distributions of $(a) v^{2}$ and $(b) B^{2}$ in case $\mathrm{Cm}$, each sampled on three spherical surfaces at indicated depths.

Turning to the magnetic spectra (Fig. 6b), we see a somewhat different picture. The magnetic energy is distributed more evenly among the largest scales: at large radii $(r=0.88 R)$ the spectra show a broad plateau up to wavenumbers $\ell \approx 30$; at intermediate radii $(r=0.50 R)$ this plateau extends to about $\ell=20$. At $r=$ $0.15 R$, the magnetic energy peaks at the largest scales $(\ell=1)$ and declines continuously toward smaller scales. There is a break at $\ell \approx 20$ to a nearly flat distribution of power with increasing wavenumber. The magnetic energy in the largest scales is actually greatest at depth, even though both the velocity amplitudes and the total magnetic energy are smaller there. This is partly in keeping with the radial variation of the mean density together with the changing scale of the flows: $\bar{\rho}$ goes from about $3.5 \mathrm{~g} \mathrm{~cm}^{-3}$ at $r=0.88 R$ to $\bar{\rho}=86$ at $0.15 R$; thus, the ratio of magnetic to kinetic energy at $\ell=1$ is roughly of order unity at all depths.

Two important points about the magnetic and velocity spectra bear emphasizing. One is that the distribution of magnetic energy as a function of scale is not given simply by equipartition with the flows at each wavenumber. On large scales the magnetic and kinetic energies are roughly comparable, whereas at small scales ME exceeds KE by up to a factor of 50. A second, related point is that the spatial distributions of the magnetic fields and flows vary appreciably with radius. Deep within the star, the magnetic field is dominated by its largest scale components, while near the surface the field is more broadly distributed in $\ell$. These radial variations in the ME spectra appear to be somewhat more than simple reflections of the depth-dependent KE spectra: rather, the ratio between $\mathrm{ME}$ and $\mathrm{KE}$ is also a function of depth, reaching its maximum at radii around $r=0.75 R$.

We caution that the power spectra presented here are likely affected by the many simplifications and limitations of our modeling. Both buoyancy driving and viscous dissipation extend here over a broad range of scales, so our simulations do not possess an extended "inertial range" in which energy could simply cascade to smaller scales. A second caveat is that our adoption of $\mathrm{Pm}>1$ is a likely contributor to the abundance of small-scale magnetic energy relative to kinetic energy; this behavior is at least expected for $\ell$ between the viscous and Ohmic diffusive scales (here $\ell>100$ ), but the amplitudes on larger scales might also be impacted. Finally, both ME and KE show much steeper declines than expected for homogeneous turbulence with or without magnetism; in most such models, $\mathrm{KE} / \ell$ and $\mathrm{ME} / \ell$ are proportional to $\ell^{-3 / 2}$ or $\ell^{-5 / 3}$ (see, e.g., Biskamp 1993; Goldreich \& Sridhar 1995; Boldyrev 2006). In this respect, the spectra are qualitatively similar to those realized in simulations of turbulent solar convection (BMT04) or A-star core convection (Browning et al. 2004). However, comparison of the spectra realized here to those expected for homogeneous, isotropic turbulence is problematic: rotation, stratification, buoyancy driving, and the artificially high viscous dissipation in our simulation all likely impact the spectral energy distribution.

\subsection{Structure and Evolution of Mean Fields}

The magnetic fields realized here clearly possess both intricate small scale structure and large-scale ordering. We turn now to an assessment of the large-scale mean fields, which we define to be the axisymmetric $(m=0)$ component of the magnetism. Many different divisions into mean and fluctuating magnetism could be employed; an axisymmetric averaging is perhaps the simplest. However defined, these large-scale fields hold particular significance in dynamo theory.

Strong axisymmetric toroidal fields are realized at many depths. These fields are displayed for three instants in the evolution of case $\mathrm{Cm}$ in Figure 7. The magnetic fields took different times to grow at the different depths. Field amplification is most rapid in the outermost regions of the star, where convection is at its most vigorous and where typical eddy sizes are smallest. Eventually, however, fields of comparable strength are established in the deep interior. The panels in Figure 7 sample $\left\langle B_{\phi}\right\rangle$ at three times: one near $t=3500$ days on the scale of Figure 3, and the others at $t=12,000$ and 14,500 days. By the time of the first snapshot, mean fields in the outermost regions had already grown to about $5 \mathrm{kG}$ in strength, but the fields deeper down were weaker by 2 orders of magnitude. In Figure $7 b$, which shows the simulation roughly 8500 days later, the mean fields at depth have grown to values comparable to those at larger radii (with typical values $\left\langle B_{\phi}\right\rangle \approx 10,000 \mathrm{G}$ ). We cannot reliably determine whether the fields at depth are generated by local dynamo action there, or are instead produced amid the more vigorous convection near the surface and then transported downwards. In both scenarios, strong fields are realized at depth only on timescales reflective of the slow overturning times deep within the star, whereas field amplification near the surface proceeds on the faster overturning time associated with the flows there.

Inspection of Figure 7 reveals that the mean fields are highly spatially nonuniform in strength, with some regions hosting very large field structures while others are more quiescent. The largest $B_{\phi}$ structures extend in radius over much of the domain, and can occupy large fractions of a hemisphere. Comparison of Figures $7 b$ and $7 c$ reveals that some of these field structures - in this case a prominent site of positive polarity in the northern hemispherepersist over intervals of thousands of days. There is still substantial field evolution - e.g., the growth of a structure of negative polarity in the southern hemisphere (Fig. 7c) - but this is most pronounced within the outer $\sim 10 \%$ of the computational domain. Once structures penetrate into the deep interior, they appear to persist in some form for timespans more reflective of the 

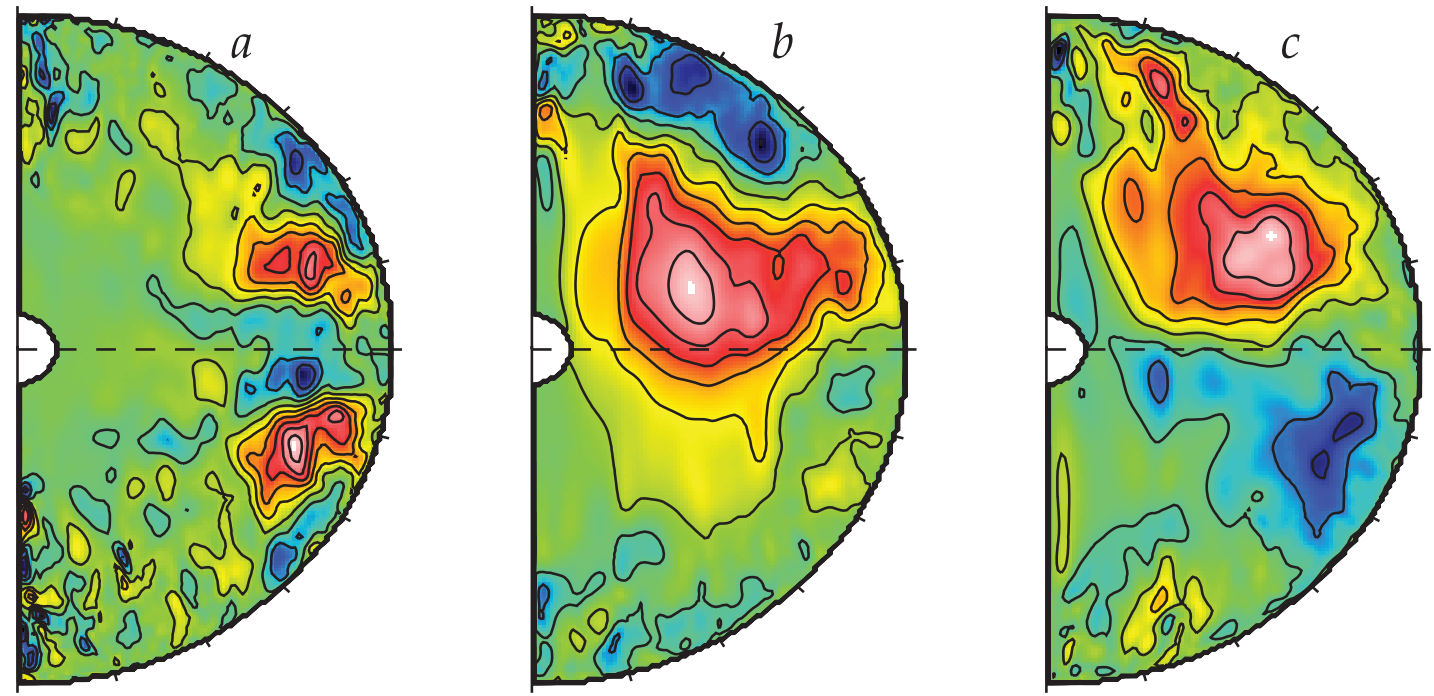

FIG. 7.- Azimuthally averaged $B_{\phi}$ as contour plots in radius and latitude at three instants in the evolution of case Cm. The three renderings sample ( $a$ ) a time prior to the saturation of the volume-averaged magnetic energy density, and times roughly $(b) 8500$ and $(c) 11,000$ days later. Polarity is indicated by the color map, with reddish tones positive (prograde polarity) and bluish tones negative (retrograde).

slow magnetic diffusion time $\left[\tau \sim L^{2} /\left(\pi^{2} \eta\right) \sim 4400\right.$ days] than of the faster convective overturning time.

The overall polarity of the fields is remarkably stable. Over the roughly $25 \mathrm{yr}$ that we have evolved simulation $\mathrm{Cm}$ after its magnetic energy equilibrated, the field near the surface has reversed its polarity - which we define as the sign of $B_{r}$ integrated over a surface in the northern hemisphere (see BMT04) - only once. This stability is in marked contrast to the frequent polarity reversals found in simulations of the solar convective envelope without a tachocline (BMT04).

\section{ESTABLISHMENT AND QUENCHING OF DIFFERENTIAL ROTATION}

Our hydrodynamical calculations (cases A, B, and C) begin in a state of uniform rotation. In all of them, however, convection quickly acts to redistribute angular momentum, ultimately establishing interior rotation profiles that vary with radius and latitude. The resulting differential rotation is partly akin to that observed at the solar surface, in that the equator rotates more rapidly than the poles; unlike the bulk of the solar convection zone, our simulations also exhibit substantial radial angular velocity contrasts, with the outer regions rotating more rapidly than the interior.

This differential rotation is assessed for the hydrodynamic case $\mathrm{C}$ in Figure 8. Shown as a contour plot is the longitudinal velocity $\hat{v}_{\phi}$, averaged in time and in longitude; rapidly rotating regions are reddish, and slower ones are bluish, all shown relative to the rotating frame. Figure $8 b$ also shows the angular velocity $\hat{\Omega}$ as a function of radius along selected latitudinal cuts. There we can see that at the surface, the overall angular velocity contrast between the equator and $60^{\circ}$ latitude is about $90 \mathrm{nHz}$, implying $\Delta \Omega / \Omega \approx 22 \%$. This is comparable to the solar angular velocity contrast $\Delta \Omega / \Omega \approx 0.25$. As in the Sun, the angular velocity decreases monotonically from equator to pole. Figure $8 b$ also reveals that $\hat{\Omega}$ generally decreases with depth, with the equator rotating about $125 \mathrm{nHz}$ faster at the top of the domain than at the bottom. Turning to the contour plot of $\hat{v}_{\phi}$, we see that the interior rotation profile is nearly constant on cylindrical lines parallel to the rotation axis. This is in keeping with the strong Taylor-Proudman constraint felt by the flows, which are heavily influenced by rotation. The angular velocity contrasts in radius and latitude are smaller in our more laminar cases A and B, but the sense of the differential rotation is the same. We have tabulated in Table 2 the contrast from equator to $60^{\circ}$ for each of these simulations.

The building of differential rotation by the rotating convective flows is not unexpected. As convective parcels rise and fall, they may be turned by Coriolis forces, yielding correlations between $v_{r}$ and $v_{\phi}$ whose effect is to transport angular momentum outward. If, on the other hand, Coriolis forces are weak (relative to buoyancy driving and pressure forces), outward-moving flows may simply tend individually to conserve angular momentum, implying an angular velocity that decreases with radius (e.g., Gilman \& Foukal 1979). The convection in our models is strongly influenced by rotation, as quantified for instance by the Rossby number Ro $=\tilde{u} /(L \Omega)$ or the convective Rossby number Roc $=$ $[\mathrm{Ra} /(\mathrm{TaPr})]^{1 / 2}$. The first of these roughly measures the strength of the Coriolis terms in equation (3) relative to the inertial ones, while the second estimates the influence of rotation compared to buoyancy driving. These are tabulated for our simulations in Table 1. In prior studies of nonlinear convection in rotating spherical shells (Gilman 1978, 1979; Brun \& Toomre 2002), a general finding has been that equatorial acceleration is realized whenever Roc is less than unity, with Coriolis forces therefore large. When Roc is large, conversely, the equatorial regions tend to rotate slower than the poles. Under strong rotational influences, angular momentum transport by the convection tends to be radially outward and latitudinally toward the equator (e.g., Brun \& Toomre 2002). The analogy in deeper spherical domains appears to be the acceleration of columns of fluid that lie far from the rotation axis, as realized here and in the core convection simulations of Browning et al. (2004). Angular momentum is globally conserved in our models, so as these regions speed up, others near the rotation axis must slow down.

The interior rotation profiles are quite different in our calculations with magnetism. Intuitively, one expects that strong magnetic fields might act like rubber bands, tying separate regions together and helping to enforce solid-body rotation. Although this analogy is simplistic, given the complex spatial and temporal structure of the magnetic fields realized here, the expectation that magnetism should lessen angular velocity contrasts turns out to be correct. In our MHD simulations, the magnetic fields react 

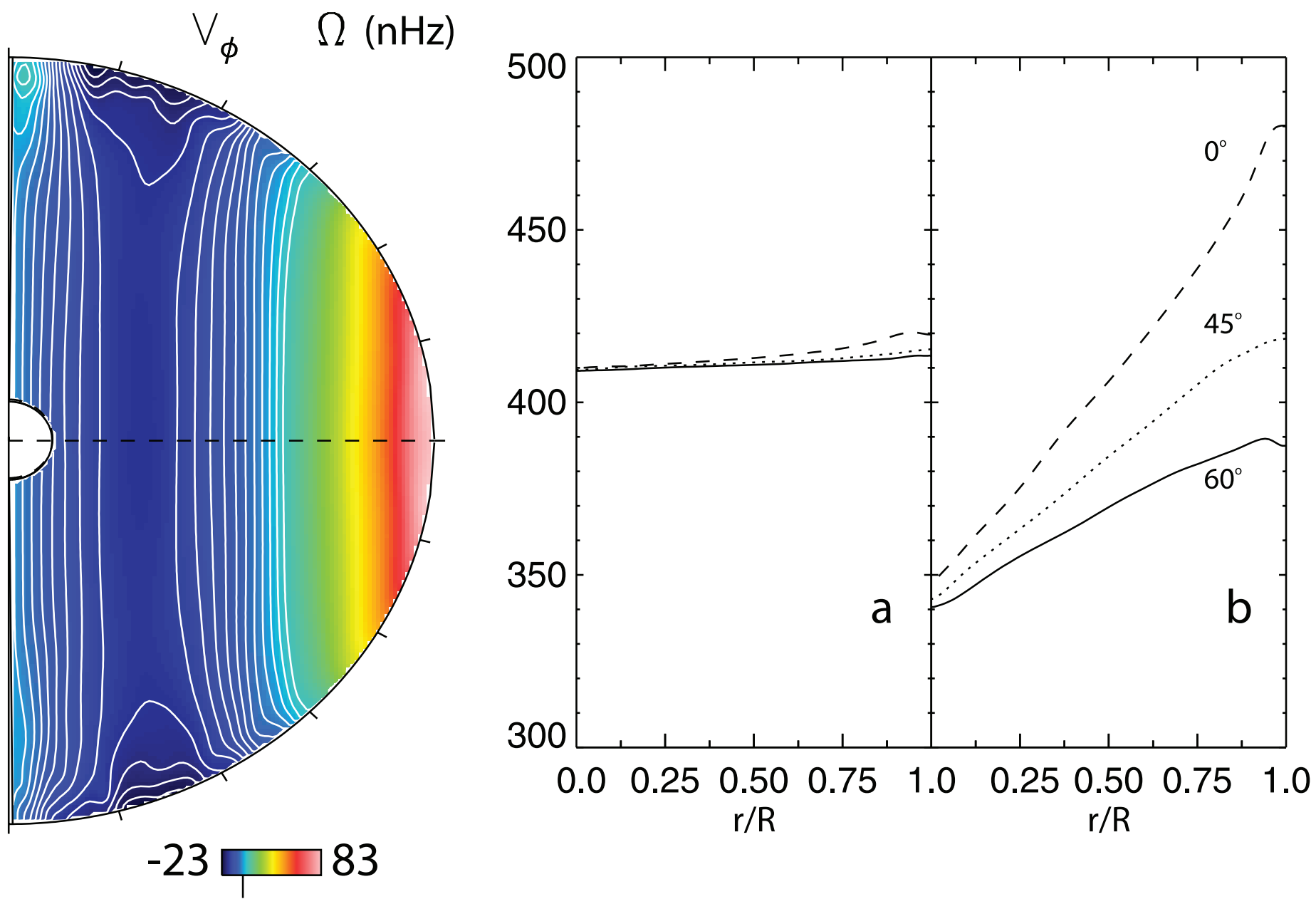

FIG. 8.-Differential rotation established in the hydrodynamic case C, and its quenching in the MHD simulation Cm. Left: Contour plot of the longitudinal velocity $\hat{v}_{\phi}$ in case $\mathrm{C}$, averaged in time and in longitude. Also displayed is the angular velocity $\hat{\Omega}$ in $(b)$ case $\mathrm{C}$ and $(a)$ case $\mathrm{Cm}$, shown as a function of radius along indicated latitudinal cuts. Case $\mathrm{Cm}$ rotates essentially as a solid body.

back strongly on the flows, acting to strongly quench the differential rotation. This behavior is assessed for case $\mathrm{Cm}$ in Figure $8 a$, which shows the angular velocity $\hat{\Omega}$ as a function of radius along cuts at various latitudes. The interior is in nearly solid body rotation; the angular velocity contrasts realized in the progenitor case C (Fig. 8b) have been almost entirely eliminated. The equator rotates less than $2 \%$ faster than the polar regions. This transition toward a uniform rotation profile is in keeping with the marked decline in DRKE noted as the magnetic fields grew in case $\mathrm{Cm}$
$(\S 4)$. In cases $\mathrm{Cm} 2$ and $\mathrm{Bm}$, which have lower equilibrated magnetic energy densities, the quenching of differential rotation is somewhat less severe. The angular velocity contrast $\Delta \Omega / \Omega$ from the equator to $60^{\circ}$ is about $8 \%$ in case Bm (compared to $14 \%$ in the hydrodynamic case B) and about $4 \%$ in case $\mathrm{Cm} 2$. Whether differential rotation is entirely, partially, or minimally quenched thus seems to be a fairly sensitive function of the magnetic energy densities realized, for ME in these three simulations differs only by a factor of about 1.4. Comparing the simulations here to

TABLE 2

Properties of Flows and Fields

\begin{tabular}{|c|c|c|c|c|c|c|}
\hline Case & A & B & $\mathrm{C}$ & $\mathrm{Bm}$ & $\mathrm{Cm}$ & $\mathrm{Cm} 2$ \\
\hline 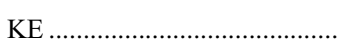 & $6.4 \times 10^{6}$ & $1.0 \times 10^{7}$ & $2.6 \times 10^{7}$ & $5.3 \times 10^{6}$ & $3.0 \times 10^{6}$ & $3.8 \times 10^{6}$ \\
\hline 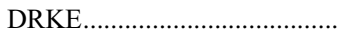 & $2.8 \times 10^{6}$ & $7.7 \times 10^{6}$ & $2.2 \times 10^{7}$ & $2.8 \times 10^{6}$ & $4.8 \times 10^{5}$ & $1.1 \times 10^{6}$ \\
\hline 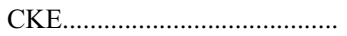 & $3.7 \times 10^{6}$ & $2.6 \times 10^{6}$ & $3.5 \times 10^{6}$ & $2.5 \times 10^{6}$ & $2.5 \times 10^{6}$ & $2.7 \times 10^{6}$ \\
\hline $\mathrm{ME} / \mathrm{KE}$ & $\ldots$ & $\ldots$ & $\ldots$ & $50 \%$ & $120 \%$ & $90 \%$ \\
\hline$\tilde{v}^{\prime}(0.94 R)$ & 24 & 22 & 23 & 19 & 19 & 19 \\
\hline$\tilde{v}^{\prime}(0.50 R)$ & 4 & 4 & 4 & 4 & 4 & 4 \\
\hline$\tilde{B}(0.94 R) \ldots \ldots \ldots \ldots \ldots \ldots \ldots \ldots \ldots \ldots \ldots \ldots \ldots \ldots$ & $\ldots$ & $\ldots$ & $\ldots$ & 2000 & 6200 & 7200 \\
\hline$\tilde{B}(0.50 R)$ & $\ldots$ & $\ldots$ & $\ldots$ & 6000 & 13100 & 10400 \\
\hline$\Delta \Omega / \Omega \ldots \ldots \ldots \ldots \ldots \ldots \ldots \ldots \ldots \ldots \ldots \ldots \ldots \ldots \ldots$ & $8 \%$ & $14 \%$ & $22 \%$ & $8 \%$ & $2 \%$ & $4 \%$ \\
\hline
\end{tabular}

Notes.-The kinetic energy density KE $\left[(1 / 2) \bar{\rho} v^{2}\right]$, averaged over volume and time, is listed along with the energy density of the convection (CKE) and the differential rotation (DRKE), together with the average magnetic energy density $\mathrm{ME}\left(B^{2} / 8 \pi\right)$ (expressed, where appropriate, as a percentage of KE). Also indicated at two depths are the fluctuating rms velocity $\tilde{v}^{\prime}\left(\mathrm{m} \mathrm{s}^{-1}\right)$ and the rms magnetic field strength $(\mathrm{G})$. Angular velocity contrast from equator to $60^{\circ}$ is indicated as percentage of overall frame rotation rate. 

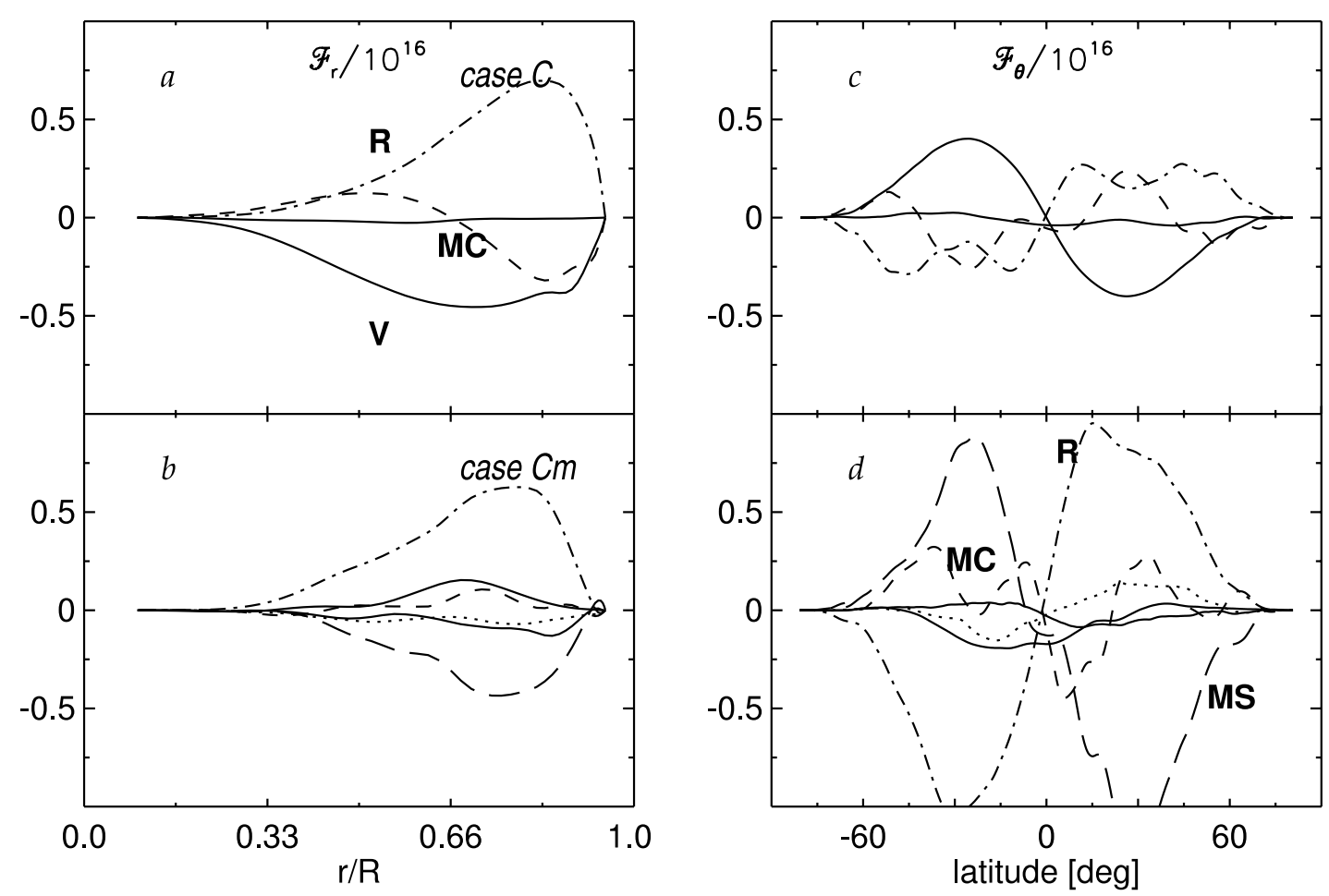

FIG. 9. - Integrated radial and latitudinal fluxes of angular momentum, averaged in time for case $\mathrm{C}$ (top) and case $\mathrm{Cm}$ (bottom). ( $a, b)$ Radial angular momentum flux $I_{r}$. $(c, d)$ Latitudinal flux $I_{\theta}$. In both left and right panels, we have indicated the contributions from Reynolds stresses (R), meridional circulations (MC), Maxwell stresses (MS), viscous diffusion (V), and large-scale magnetic torques (MT), together with their sum (solid line). Positive quantities represent fluxes radially outward or directed latitudinally from north to south.

those of BBT05, BMT04, and Browning et al. (2006) reinforces the view that differential rotation can persist when magnetism is weak relative to the flows (with $\mathrm{ME} / \mathrm{KE}$ less than about $30 \%$ ), is partially quenched for intermediate-strength fields (with cyclical feedbacks between the magnetism and differential rotation sometimes realized), and is strongly suppressed whenever the magnetism is very strong (equipartition-strength or greater). Our simulations show that for sufficiently turbulent flows, such equipartition-strength magnetic fields can be realized even in fully convective stars, and sustained once the differential rotation has been eliminated.

In the following section we examine the manner in which these zonal flows are established in the hydrodynamic simulations and quenched in the presence of strong magnetic fields.

\subsection{Angular Momentum Transport}

The interior rotation profiles realized here result from a variety of competing processes: angular momentum can be redistributed by Reynolds stresses, by meridional circulations, by viscous diffusion, or by torques and Maxwell stresses associated with the magnetic fields. No simple analytical tools allow us to reliably predict how each of these effects will act, and how they will combine to shape the interior rotation profile. But the present simulations offer an opportunity to assess these processes "after the fact": we can constrain the angular momentum transport afforded by each and see how, together, they yield differential rotation in the hydrodynamic cases and weaker angular velocity contrasts in the MHD simulations.

To analyze the angular momentum transport, we turn (in the manner of Elliott et al. 2000; BMT04) to the zonal component of the momentum equation, expressed in conservative form and averaged in time and in longitude:

$$
\frac{1}{r^{2}} \frac{\partial\left(r^{2} \mathcal{F}_{r}\right)}{\partial r}+\frac{1}{r \sin \theta} \frac{\partial\left(\sin \theta \mathcal{F}_{\theta}\right)}{\partial \theta}=0,
$$

where

$$
\begin{aligned}
\mathcal{F}_{r}= & \bar{\rho} r \sin \theta\left[-\nu r \frac{\partial}{\partial r}\left(\frac{\hat{v}_{\phi}}{r}\right)+\widehat{v_{r}^{\prime} v_{\phi}^{\prime}}+\hat{v}_{r}\left(\hat{v}_{\phi}+\Omega r \sin \theta\right)\right. \\
& \left.-\frac{1}{4 \pi \bar{\rho}} \widehat{B_{r}^{\prime} B_{\phi}^{\prime}}-\frac{1}{4 \pi \bar{\rho}} \hat{B}_{r} \hat{B}_{\phi}\right]
\end{aligned}
$$

and

$$
\begin{aligned}
\mathcal{F}_{\theta}= & \bar{\rho} r \sin \theta\left[-\nu \frac{\sin \theta}{r} \frac{\partial}{\partial \theta}\left(\frac{\hat{v}_{\phi}}{\sin \theta}\right)+\widehat{v_{\theta}^{\prime} v_{\phi}^{\prime}}\right. \\
& \left.+\hat{v}_{\theta}\left(\hat{v}_{\phi}+\Omega r \sin \theta\right)-\frac{1}{4 \pi \bar{\rho}} \widehat{B_{\theta}^{\prime} B_{\phi}^{\prime}}-\frac{1}{4 \pi \bar{\rho}} \hat{B}_{\theta} \hat{B}_{\phi}\right]
\end{aligned}
$$

are the mean radial and latitudinal angular momentum fluxes, respectively. The terms on the right hand side of equations (21) and (22) are, in order, the contributions from viscous diffusion, Reynolds stresses, meridional circulations, Maxwell stresses, and large-scale magnetic torques. The Reynolds stresses are associated with correlations of the fluctuating velocity components $\left(v_{r}^{\prime}, v_{\theta}^{\prime}\right.$, and $\left.v_{\phi}^{\prime}\right)$ that arise when the convective structures possess organized tilts. Similarly, Maxwell stresses are correlations of the fluctuating magnetic field components that correspond to the tilt and twist of magnetic structures.

To more easily analyze the various components of $\mathcal{F}_{r}$ and $\mathcal{F}_{\theta}$, we integrate over colatitude and radius to find the net fluxes through shells at each radius and through cones at each latitude:

$$
\begin{aligned}
I_{r}(r) & =\int_{0}^{\pi} \mathcal{F}_{r}(r, \theta) r^{2} \sin \theta d \theta \\
I_{\theta}(\theta) & =\int_{r_{\text {bot }}}^{r_{\text {top }}} \mathcal{F}_{\theta}(r, \theta) r \sin \theta d r .
\end{aligned}
$$


These are displayed for cases $\mathrm{C}$ and $\mathrm{Cm}$ in Figure 9. We have identified there the contributions from Reynolds stresses (denoted R), meridional circulations (MC), viscous diffusion (V), Maxwell stresses (MS), and large-scale magnetic torques (MT). In constructing Figure 9, we averaged the fluxes over about 150 days of evolution.

Turning first to the integrated radial angular momentum flux in case C (Fig. 9a), we see that the Reynolds stresses act to transport angular momentum radially outwards. They are opposed mainly by viscous diffusion, which transports angular momentum inwards at all radii; the flux associated with meridional circulations plays a smaller role here, but acts to transport angular momentum to middepth $(r \approx 0.66 R)$ from either smaller or larger radii. The total flux (solid line) is nearly zero, confirming that the rotation profile is well equilibrated. Note that the steady-state inward transport due to viscous diffusion sampled here is consistent with the prevailing differential rotation in case $\mathrm{C}$ : the viscous flux is negative whenever $\partial / \partial r\left(\hat{v}_{\phi} / r\right)$ is positive, as it is in case $\mathrm{C}$.

The latitudinal angular momentum flux in case $\mathrm{C}$, sampled in Figure $9 c$, conveys a similar picture. There the Reynolds stresses act to transport angular momentum toward the equator, since the associated flux is positive in the northern hemisphere and negative in the southern. Again, viscous diffusion acts in the opposite manner, transporting angular momentum poleward. The meridional circulations figure more prominently than they did in the radial balance: here they act mainly in concert with the Reynolds stresses.

Examining the angular momentum transport in case $\mathrm{Cm}$ (Figs. $9 b$ and $9 d$ ) reveals that magnetic fields can play a major role in establishing the interior rotation profile. Figure $9 b$ shows that strong Maxwell stresses are realized throughout much of the interior, and that they tend to transport angular momentum inwards. That the Reynolds and Maxwell stresses transport angular momentum in opposite directions is understandable: the corresponding terms in equations (20) and (21) carry opposite signs, so as long as correlations between fluctuating magnetic field components $\left(B_{r}^{\prime} B_{\phi}^{\prime}\right)$ are in the same sense as the corresponding velocity correlations $\left(\widehat{v_{r}^{\prime} v_{\phi}^{\prime}}\right)$, angular momentum transport by Maxwell stresses will oppose that of the Reynolds stresses. The Reynolds stresses did not grow to compensate for the inward-directed flux due to Maxwell stresses; thus, the region of prograde flow at large radii (realized in case $\mathrm{C}$ ) was gradually slowed, yielding the nearly solid body rotation profile of case $\mathrm{Cm}$. In the equilibrated state sampled by Figure $9 b$, the viscous flux of angular momentum is also much smaller than it was in case C (Fig. 9a). This is a result, rather than a cause, of the nearly solid body rotation profile established in case $\mathrm{Cm}$ : the viscous transport term is proportional to $-\partial / \partial r\left(\hat{v}_{\phi} / r\right)$, so small gradients of the angular velocity imply a vanishing viscous transport of angular momentum. In cases Bm and $\mathrm{Cm} 2$, which have weaker magnetic fields, the Maxwell stresses act in the same sense, but are weaker and so do not have as great an impact on the differential rotation.

The latitudinal transport in case Cm, sampled in Figure $9 d$, tells a similar story. The dominant balance is between equatorward transport by the Reynolds stresses and poleward transport by Maxwell stresses. The angular momentum fluxes due to viscous diffusion, meridional circulation, and large-scale magnetic torques are all smaller. Somewhat surprisingly, the large-scale magnetic torques, although small, generally oppose the Maxwell stresses associated with the fluctuating fields, implying that correlations of the form $\hat{B}_{\theta} \hat{B}_{\phi}$ and $\overline{B_{\theta}^{\prime} B_{\phi}^{\prime}}$ are of the opposite sign.

Taken together, these analyses yield some insight into how differential rotation is established in hydrodynamic cases and quenched in MHD ones. In the parameter regime probed here, the Reynolds stresses associated with the turbulent convection tend to transport angular momentum radially outward and latitudinally toward the equator. In the hydrodynamic cases, this transport is opposed only by viscous diffusion and, to some extent, meridional circulations; the result is an acceleration of regions at large radii and low latitudes, until the steady state sampled by Figures $9 a$ and $9 c$ (and by the contour plot of Fig. 8) is reached. In the MHD cases, however, the Reynolds stresses must also counteract the effect of Maxwell stresses, which tend to transport angular momentum poleward and inward. The meridional circulations and large-scale magnetic torques do not adjust to cancel out the effect of these Maxwell stresses, so the net result is a lessening of the differential rotation.

This general picture appears robust, but we caution that some of the detailed features of Figure 9, e.g., the exact magnitude of the viscous flux, depend in a highly nonlinear fashion on each other and on other attributes of the simulation. The viscous flux, for instance, depends on the overall angular velocity gradients that are established - but it is in part responsible for setting those angular velocity gradients, through its competition with the Reynolds stesses, meridional circulations, etc. Thus, viscous transport is a major player in the angular momentum balance in case $\mathrm{C}$, but a negligible one in the evolved state of case $\mathrm{Cm}$ : it did not vanish because of the presence of magnetic fields, but gradually tapered away as the Maxwell stresses lessened the angular velocity contrast. We have chosen for simplicity to show only the steady-state fluxes of angular momentum in both cases; during the initial transient phases in which the rotation profiles are established, the sense of the fluxes-i.e., which ones sought to speed up the equator and which to slow it - was generally the same as that described here.

\section{ROTATION, HELICITY, AND THE GENERATION OF FIELDS}

The magnetic fields realized here possess several striking properties. Although substantial magnetic energy is realized on small scales, there is also some order on the largest scales. Strong $(\sim 10 \mathrm{kG})$ axisymmetric toroidal fields are generated by the dynamo, and account for up to $20 \%$ of the magnetic energy at some sites. Some of these strong large-scale field structures persist for thousands of days; the overall polarity of the field in case $\mathrm{Cm}$, our longest evolved simulation, has flipped only once in about $30 \mathrm{yr}$ of simulated evolution. Furthermore, these global field structures can be realized without the aid of stretching by differential rotation, for the interiors of our most turbulent MHD simulations rotate nearly as solid bodies.

These results stand in sharp contrast to those of some prior simulations of convection in more massive stars. In computational models of the solar convective envelope, BBT05 found that the toroidal mean magnetic energy was typically less than $5 \%$ of the total ME; the polarity of the mean field typically reversed at chaotic intervals of less than 600 days. In simulations that also included the tachocline below (Browning et al. 2006), higher values of TME/ME were achieved only amid the strong shear of the tachocline itself; the polarity evolution of the fields was stabilized by the presence of strong mean fields in the radiative layer. In neither of these sets of simulations did the magnetic energy become strong enough to quench the differential rotation entirely. Similarly, models of dynamo action in the convective cores of A-type stars (BBT05) indicated that strong large-scale fields were mostly realized in the shearing layer near the core-envelope boundary. Those simulations also exhibited a rich variety of interactions between the magnetism and the differential rotation: angular velocity contrasts were almost 


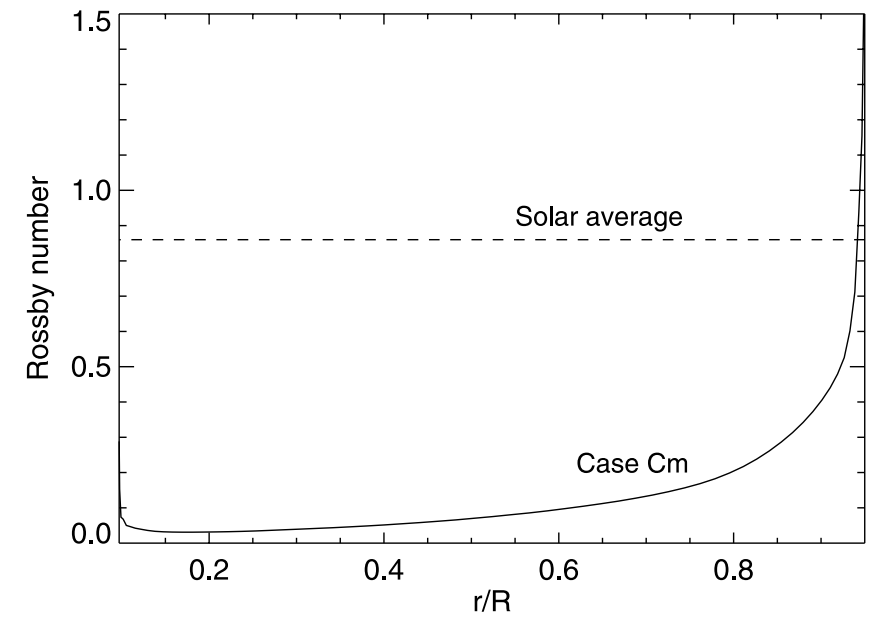

FIG. 10.- Local Rossby number Ro $=(\nabla \times u) / 2 \Omega$, averaged in time and on spherical surfaces. Also shown is the average value of Ro $\approx 0.86$ within the solar convection zone simulations of Browning et al. (2006). The lower Rossby numbers realized here indicate a stronger rotational influence.

entirely eliminated in rapidly rotating cases, while slower rotators showed cyclical feedbacks between differential rotation and magnetism.

Some guidance in interpreting these results may be afforded by findings from mean field theories (MFTs) of the dynamo process. In many such theories, a key role is played by the kinetic helicity, $\boldsymbol{v} \cdot \nabla \times \boldsymbol{v}$, which is related to the twisting and winding of the convective flows (see Moffatt \& Tsinober 1992). Under a number of simplifying assumptions, it can be shown that the " $\alpha$-effect" of traditional MFT is proportional to the kinetic helicity of the turbulence, implying that more helical flows might build strong large-scale flows (e.g., Parker 1955; Steenbeck et al. 1966; Moffatt 1978; Moffatt \& Proctor 1982; Brandenburg \& Subramanian 2005). This general expectation has been partly born out by simulations of dynamo action in forced helical turbulence and by numerical calculations using turbulent closure schemes (e.g., Blackman 2003a; Pouquet \& Patterson 1978). In such modeling, the spectrum of the magnetic fields realized by dynamo action changed as the kinetic helicity was varied, with more large-scale field generated when the helicity was increased. A common expectation is thus that flows without helicity can act as dynamos, but the fields are typically on the scale of the turbulent eddies (e.g., Brandenburg \& Subramanian 2005). More recent numerical modeling has suggested, however, that the linkages between kinetic helicity and large-scale magnetic fields may not be so clear-e.g., Courvoisier et al. (2006) found no relation between the $\alpha$-effect and kinetic helicity in models of a variety of chaotic flows.

The convective flows in our simulation are strongly influenced by rotation. The local Rossby number $\mathrm{Ro}_{w}=\nabla \times \boldsymbol{v} / 2 \Omega$ (which compares the fluid vorticity to the "planetary" vorticity $2 \Omega$ ) is less than 0.1 throughout most of the interior; its radial variation for case $\mathrm{Cm}$ is shown in Figure 10. Also shown for comparison there is the globally averaged value of $\mathrm{Ro}_{w} \approx 0.86$ for the penetrative solar dynamo calculations of Browning et al. (2006) (calculated within the bulk of the convection zone). The local Rossby number here is significantly less than in the solar simulation, even though the angular velocity in case $\mathrm{Cm}$ is equal to the solar rate of $\Omega=2.6 \times 10^{-6} \mathrm{~s}^{-1}$. Likewise, the global estimates of Ro and Roc given in Table 2 are significantly lower than those calculated for the solar simulations of BMT04 or Browning et al. (2006). The influence of rotation is stronger here for the same rotation rate because the stellar luminosity is much lower, so typical convective velocities are slower than in the solar case, and the convective overturning time is longer. Flows that take more than one rotation period to overturn can be strongly affected by Coriolis forces, whereas those that overturn faster cannot; here only the rapid, small-scale flows near the surface have overturning times that approach the rotation period. Note that the rotation rate adopted here is lower than that observed for most mid-late M stars, so nearly all such stars are probably strongly influenced by rotation.

The general trend that emerges from comparing our simulations to prior ones is that a stronger rotational influence, and hence lower Ro, implies both higher magnetic energy densities relative to kinetic, and magnetic fields of increasingly large spatial scale. When Ro is close to unity, as in the Sun (Browning et al. 2006; BBT05), the magnetic energy generally appears to be small enough that differential rotation can readily persist. Under somewhat stronger rotational influences, as realized in some of the A-star core dynamos of BBT05, the differential rotation and magnetism may feed back on each other, possibly yielding cyclical waxing and waning of field strength. At the still stronger rotational influences sampled here and in the more rapidly rotating cases of BBT05, the helical convective flows are able to build magnetism of equipartition strength without the aid of differential rotation; angular velocity contrasts realized in hydrodynamic cases are greatly reduced by the strong magnetic fields.

Simulations of the geodynamo also lend support to the idea that stronger rotational influence can lead to magnetic fields on larger spatial scales. Christensen \& Aubert (2006) found that lower Ro led to magnetic fields with a larger "dipole fraction," defined as the power in the $\ell=1$ mode divided by that in modes $\ell=1-12$. They and others have suggested that predominantly dipolar fields are realized when inertial forces are small relative to Coriolis forces (see also Sreenivasan \& Jones 2006; Olson \& Christensen 2006). The transition between dipolar and multipolar fields in their models occurred at a "modified Rossby number" $\mathrm{Ro}_{l} \approx 0.1$, where $\operatorname{Ro}_{l}=\operatorname{Ro}\left(\ell_{u} / \pi\right)$, with $\ell_{u}$ the mean spherical harmonic degree of the flows. Constructing a local $\mathrm{Ro}_{l}$ as a function of depth in the simulations here (using the power spectra in $\S 5.2$ ) suggests that our models may be on the cusp of entering into the predominantly dipolar regime identified by Christensen \& Aubert (2006). At most depths, $\mathrm{Ro}_{l}$ in our simulations is still somewhat greater than 0.1 , and the dipole fraction is low; deep in the interior, however, $\mathrm{Ro}_{l} \approx 0.1$, and the local dipole fraction rises to about $30 \%$. These results are suggestive of the role that rotation may play in setting the field geometry, although clearly much more work is needed to clarify how stratification, dynamo supercriticality, and other effects might likewise enter into the field strength and morphology.

Quantifying the connection between rotational influence (as measured by Ro) and kinetic helicity, which figures so prominently in MFT, is a complex task. The two are clearly related, for it is partly the overall rotation that imparts a global ordering to the helicity: downflows in the northern hemisphere tend to contract, and because of Coriolis forces rotate counterclockwise, implying anticyclonic vorticity (e.g., Miesch et al. 2000). Thus, on average the kinetic helicity is negative in the northern hemisphere and positive in the southern hemisphere. A naive expectation might therefore be that as Coriolis forces become increasingly important, this process would lead to stronger net helicity. Indeed, in simple models, the average kinetic helicity is often taken to be proportional to the overall angular velocity (e.g., Durney et al.1981; Noyes et al. 1984), reflecting the ability of Coriolis forces to twist convective parcels as they rise or descend. Under 
such an approximation, the "dynamo number" of MFT may then be proportional to the angular velocity squared (e.g., Noyes et al. 1984). Such direct connections between helicity and rotation rate are not realized in our simulations. Both the azimuthally averaged kinetic helicity and its rms values are smaller here than in, for instance, the solar calculations of Browning et al. (2006), even though Ro is substantially lower. This probably reflects several key differences between the flows here and in the solar simulations: the velocities here are lower, and the stratification weaker throughout most of the interior, leading to less asymmetry between upflows and downflows, and hence less preference for one sign of helicity. Furthermore, the convection here shows some tendency to align in rolls parallel to the rotation axis, reflecting the strong Taylor-Proudman constraint; in such rolls, the velocity is mostly perpendicular to the rotation axis, but the vorticity is mostly parallel to it, resulting in a small average kinetic helicity (e.g., Knobloch et al. 1981). A further complication is that recent numerical modeling and asymptotic analysis of unstratified turbulence has shown that a preference for one sign of vorticity, and hence a high net helicity, is established at moderate rotation rates with $\mathrm{Ro} \approx 0.1 \rightarrow 1$, but eliminated as rotation becomes even more rapid (Sprague et al. 2006). This results in little net helicity in the most rapid rotators. Whether such analysis is relevant to the stratified flows here is not clear at this stage, but bears further study.

Drawing the further connection between kinetic helicity and the generation of large-scale magnetic fields in our simulations appears to be even more difficult. Like Livermore et al. (2007), we have seen no clear linkages between the magnitude or power spectrum of the helicity and that of the magnetic field. Indeed, the power spectrum of this quantity in the present simulations is qualitatively similar to one constructed for the convective envelope in the solar simulations of Browning et al. (2006). As noted above, the magnitude of the kinetic helicity is smaller here than in those simulations or the ones of BBT05, yet the axisymmetric magnetic field is stronger. More sophisticated theories relating the growth of fields to the kinetic helicity appear required; alternatively, it may be that the dynamo action at some scales could be partly "quenched" by the growth of current helicity at those scales, as suggested in some variants of MFT (see, e.g., Blackman 2003b). Much further work will be required to assess whether such theories or variations thereof can accurately describe the growth of fields in the complex flows here.

We close this section with brief comments on the strength and temporal variability of the fields realized here. That mean toroidal fields of order $10 \mathrm{kG}$ strength can be maintained in a fully convective star for long periods of time might come as a surprise: in the solar convective envelope, simple estimates suggest that fields of that strength would quickly rise due to magnetic buoyancy (Parker 1975). Indeed, the rapid timescale for such rise was a major factor in identifying the stable layer as the likely seat of the global solar dynamo. Here, however, the limits on field strength imposed by magnetic buoyancy are less severe. A crude estimate of the upward velocity for a buoyant flux tube in an unstably stratified layer is

$$
u \sim v_{a}\left(\frac{\pi a}{C_{d} \Lambda}\right)
$$

with $v_{a}=B /(4 \pi \rho)^{1 / 2}$ the Alfvén speed, $C_{d}$ the aerodynamic drag coefficient, $a$ the tube radius, and $\Lambda$ the pressure scale height (Parker 1975). For constant $a / \Lambda$ and $C_{d}$, this estimate implies that the characteristic timescale for fields to rise from the center of an M dwarf to its surface is about a factor of 10 longer than the time needed for fields to rise through the solar convective envelope. The main difference is that the density in the $\mathrm{M}$ star is substantially greater, so the Alfvén speed is lower and the rise time greater. Finally, we note that the long temporal stability of the field polarity here is also striking, but may partly reflect the lower typical convective velocities realized here. If the field is modeled as a collection of overlapping dipole moments corresponding to typical convective eddies, and these individual eddies are uncorrelated, then the overall magnetic dipole should evolve on a random-walk timescale, $\tau_{B} \sim R^{2} / \nu_{t}$, with $\nu_{t} \sim v_{c} l_{\text {ed }}$ (Thompson \& Duncan 1993). This estimate would imply $\tau_{B} \sim 6$ yr for motions near the surface. The field actually appears to evolve on somewhat slower timescales that are more characteristic of the flows deep in the interior. A possibly analogous effect was found in Browning et al. (2006), where the presence of organized mean fields deep in the interior served to largely stabilize the sense of fields produced in the convection zone. This is a cautionary tale: the field dynamics near the stellar surface may well reflect couplings with an interior field that is hidden from view.

\section{CONCLUSIONS AND PERSPECTIVES}

We have presented global 3D MHD simulations of the interiors of fully convective $\mathrm{M}$ stars rotating at the solar angular velocity. These nonlinear simulations attempt to model a 0.3 solar-mass star with reasonable fidelity: the stratifications of density and temperature are consistent with those of a 1D stellar model, as are the luminosity and rotation rate. Although many great simplifications have been made, we believe that several of the conclusions of our work may prove to be robust. We reiterate these principal findings below, and comment briefly on the uncertainties associated with each. We also compare our work to the simulations of DSB06, and to the limited observational constraints presently available.

The convection realized in these simulations is characterized by small-scale, intermittent flows near the stellar surface, and by weaker, large-scale flows in the deep interior. The radial variation in the size of typical convective eddies is driven mainly by the strong density stratification (with $\rho_{\text {bot }} \approx 100 \rho_{\text {top }}$ ). The weakening of convective velocities at depth arises partly because the luminosity $L_{c}$ that must be carried by the convection-essentially the difference between the total luminosity $L_{t}$ and the radiative luminosity $L_{r}$ - is quite small there, even though the star is everywhere unstably stratified. Thus, in the cores of our model stars, convection is fairly weak and radiation actually still transports much of the energy. The relatively modest energy transport afforded by convection in the deep interior does not result directly from convective suppression by magnetic fields: convection is weak at depth even in hydrodynamic calculations.

The flows act as a magnetic dynamo, amplifying a small seed field by orders of magnitude and sustaining it against Ohmic decay. The equilibrated magnetic energy density in our highest resolution, most turbulent simulation $\mathrm{Cm}$ is roughly $120 \%$ of the kinetic energy density relative to the rotating frame. The resulting magnetic fields possess structure on a wide variety of spatial scales; the typical size of field structures is largest in the deep interior, and smaller near the surface. Strikingly, the magnetic field possesses a strong axisymmetric mean component, with the toroidal mean energy accounting for up to $20 \%$ of the total magnetic energy. Such prominent large-scale mean fields have not been realized in simulations of the solar convection zone (BMT04), except within a stably stratified tachocline of shear (Browning et al. 2006). The mean fields realized here also 
possess remarkably stable polarities: only one reversal of the overall polarity was realized in the roughly $25 \mathrm{yr}$ evolution of case $\mathrm{Cm}$. This result, too, stands in contrast to prior simulations of solar-like convection in spherical shells (BMT04), in which the overall polarity tended to flip at irregular intervals of less than 600 days.

Differential rotation is established by the convection in hydrodynamic cases, but reduced in MHD simulations. In our most turbulent case, $\mathrm{Cm}$, which has the strongest dynamo-generated magnetic fields, the differential rotation of the hydrodynamic progenitor is almost entirely eliminated. This occurs because of strong Maxwell stresses, which tend to oppose the equatorward transport of angular momentum by Reynolds stresses. In the nonmagnetic cases, the differential rotation is solar-like at the surface, with a fast equator and slow poles; the interior rotation profile is largely constant on cylinders, in accord with the strong Taylor-Proudman constraint. The angular velocity contrasts in those hydrodynamic cases are fairly modest, in keeping with simulations of rapidly rotating solar-like stars (B. P. Brown et al. 2008, in preparation); it is possible that this reflects nonmagnetic mechanisms for quenching zonal flows in rapidly rotating systems, as discussed in mean-field models of angular momentum transport (e.g., Kitchatinov \& Ruediger 1995). No cyclical feedbacks between the differential rotation and the magnetic field-noted in prior simulations of core convection in A-type stars at certain rotation rates (BBT05) - are seen in case $\mathrm{Cm}$. Rather, equipartition-strength fields are sustained even in the absence of any differential rotation. More interplay between the differential rotation and magnetic fields is realized in the two cases $\mathrm{Bm}$ and $\mathrm{Cm} 2$, in which the magnetism is weaker relative to the kinetic energy and the differential rotation is somewhat more persistent. The magnetic fields also impact the convective (nonaxisymmetric) flows, although much less drastically; this weakening of the convection may be somewhat akin to that explored by Chabrier et al. (2007) in the context of mixing-length theory.

We have argued in $\S 7$ that several key attributes of these simulations - the strength of the magnetic fields realized, their strong large-scale axisymmetric components, and the quenching of differential rotation-may depend crucially on the influence of rotation. Although the simulations here rotate at the solar angular velocity, the rotational influence on the convective flows is far stronger than in simulations of the solar convective envelope (e.g., Browning et al. 2006) or the cores of A-type stars rotating at the same angular velocity (BBT05). Here, the very slow convective flows imply Rossby numbers significantly smaller than in more luminous stars at the same rotation rate. A comparison of our simulations here to others at varying Rossby number (BBT05; Browning et al. 2006) suggests that as the rotational influence becomes stronger, the magnetic energy density grows larger relative to the kinetic energy density. When the ratio $\mathrm{ME} / \mathrm{KE}$ is small $(<30 \%)$, strong differential rotation can persist; as ME grows larger (with increasing rotational influence), a regime may be reached in which the differential rotation and $\mathrm{ME}$ each undergo cyclical waxing and waning. Finally, in stars where rotation is still more important-as achieved in the $4 \Omega$. cases of BBT05 and in our simulations $\mathrm{Cm}$ and $\mathrm{Cm} 2$ here-ME can exceed KE without the aid of differential rotation, and any persistent angular velocity contrasts are strongly quenched. These conclusions are also tentatively born out by preliminary simulations of dynamo action in more rapidly rotating solar-like stars (B. Brown et al. 2008, in preparation).

Thus, we think it likely that had the simulations here been rotating at only a quarter of the solar angular velocity (to yield
Rossby numbers more in accord with the $1 \Omega_{\odot}$ simulations of BBT05), cyclical feedbacks of ME on DRKE would have been obtained even in our most turbulent cases. At still slower rotation rates, the differential rotation established in hydrodynamic cases would likely have persisted in the presence of the weaker sustained magnetism. But the simulations here already correspond to rotational velocities below what can presently be measured: for a star with a radius of $2 \times 10^{10} \mathrm{~cm}$, the solar angular velocity of $\Omega_{\odot}=2.6 \times 10^{-6} \mathrm{~s}^{-1}$ implies $v_{\text {rot }} \approx 0.5 \mathrm{~km} \mathrm{~s}^{-1}$, below current detection limits of $v \sin i \approx 2 \mathrm{~km} \mathrm{~s}^{-1}$ (e.g., Delfosse et al. 1998). From the point of view of observations, even the models presented here would be "nonrotating."

Like all those who would simulate stellar convection, we have made major simplifications in our modeling. Of these, our use of effective eddy viscosities and diffusivities - which are vastly greater than their microscopic counterparts in actual stars-is arguably the most severe. Other potentially important simplifications include our omission of the inner and outer few percent of the stellar interior (our computational domain extends from $0.08 R$ to $0.96 R$ ), our adoption of a perfect gas equation of state, and the limited duration of the simulations compared to the very long thermal relaxation timescales in actual stars. It is difficult to estimate the impacts each of these may have, but we are encouraged by the reasonable success that similar simulations of the solar interior have enjoyed in matching the detailed observational constraints provided by helioseismology (Miesch et al. 2006; Browning et al. 2006).

Probably the greatest uncertainty associated with these simplifications is the extent to which changes in other simulation parameters - e.g., the magnetic Reynolds and Prandtl numberscould mimic the effects of rotation on magnetic field strength and differential rotation. Both the strength and morphology of the field are likely sensitive to these parameters at some level, as indicated by the modest differences in ME and DRKE between our three cases $\mathrm{Cm}, \mathrm{Cm} 2$, and $\mathrm{Bm}$. Our simulations were conducted in an $\mathrm{Rm}, \mathrm{Pm}$ regime probed frequently by prior simulations, and yet they exhibit quite distinct behavior. The closest analogs in our own prior work are the most rapidly rotating cases of BBT05; this fact, and a comparison to cases at other Rossby numbers, is a partial motivation for our suggestion that the Rossby number is a dominant control parameter in determining the strength and geometry of the magnetic fields. Future work that more thoroughly probes the parameter space of rotation rate, $\mathrm{Rm}$, and $\mathrm{Pm}$ will be needed in order to put this tentative suggestion on firmer ground.

Our results partly agree with those of DSB06, in that we find that strong, equipartition-strength magnetic fields can be generated at certain rotation rates. Like them, we also find that the fields have substantial axisymmetric mean components, and that they possess structure on both large and small spatial scales. The most significant differences between our findings and theirs concern the differential rotation established in the interior: they find that hydrodynamic simulations establish an antisolar differential rotation, and that this differential rotation persists even when strong magnetism is present. We find solar-like differential rotation in our hydrodynamical models, and nearly solid body rotation in our most turbulent MHD simulations. Although the origins of this discrepancy in our results are not certain, it may be partly caused by the differing strengths of rotation and turbulence in our simulations compared to those of DSB06. Although the rotational velocities adopted in DSB06 are far greater than in actual stars, so are the convective velocities (because the stellar luminosity was greatly enhanced). It is difficult to say how this rescaling affects the delicate balance of convection, rotation, and 
magnetism, but it is reasonable to suggest that the overall rotational influence in most of their simulations is somewhat smaller than in ours. A crude estimate of the rotational influence is given by $\mathrm{Ro} \approx\left(u_{\mathrm{rms}} / R\right) / 2 \Omega$; for most of their simulations, Ro is greater than in ours, indicating that rotation is weaker relative to inertia. Only for their two most rapidly rotating cases does Ro drop below the values reported for our cases. They note a trend toward increasing $\mathrm{ME} / \mathrm{KE}$ with increasing rotation rate in their simulations; they also note that the overall angular velocity contrast is reduced as the rotation rate is increased (in their magnetic cases). Thus, we suspect that for somewhat more rapid rotation, they too would find even stronger quenching of the differential rotation, perhaps yielding solid-body rotation like that realized in our case $\mathrm{Cm}$. Alternatively, at fixed rotation rate, more turbulent flow (and higher Rm and Re) may lead to stronger magnetic energy densitites (as evinced by a comparison of case Bm to case $\mathrm{Cm}$ ), and hence to stronger quenching of the differential rotation. Indeed, our case $\mathrm{Cm}$ has a somewhat higher Re (and significantly higher Rm) than the simulations of DSB06 that have comparable rotation rates; our more laminar case $\mathrm{Bm}$ possesses magnetic energy densities and angular velocity contrasts somewhat more akin to those of DSB06. Other differences between our simulations and those of DSB06 also impact the results in subtler ways. For instance, the morphology of the convective flows and their variation with radius are strongly affected by the density stratification; our overall density contrast of $\approx 100$ is consistent with a 1D stellar model, whereas theirs is a factor of 20 smaller, so naturally the developed flow patterns in our simulations differ somewhat. Other differences-e.g., the boundary conditions adopted on the flow fields - may also impact the results at some level.

If we are correct in suggesting that rotational influence is the dominant control parameter in setting the magnetic energy, the field morphology, and (indirectly, through the feedback of Maxwell stresses) the differential rotation, then some straightforward observational predictions follow. Rapidly rotating M stars should generally show strong magnetic fields and little or no differential rotation. Less rapidly rotating ones may show cyclical magnetic energy and differential rotation, while the slowest rotators are most likely to harbor persistent angular velocity contrasts, accompanied by somewhat weaker average magnetic energies. Again extrapolating from our limited probing of parameter space, the axisymmetric component of the field should account for a greater fraction of the magnetic energy in progressively more rapid rotators. Although the observational constraints on magnetic fields and differential rotation in fully convective $\mathrm{M}$ stars are still scarce, it appears that these predictions are at least consistent with what has so far been observed. Donati et al. (2006) reported that v374 Peg had a strong, mostly axisymmetric magnetic field, with no evident differential rotation. Their target star was very rapidly rotating, in keeping with our suggestion that differential rotation should be strongly quenched in such stars. At still lower masses, Reiners \& Basri (2007) have found that magnetic activity is detectable even in some stars that are not detectably rotating; such stars may conceivably have low enough convective velocities that even rotation rates below the observational detection limit could imply a reasonably strong rotational influence and hence lead to vigorous dynamo action. Much more work will be required to elucidate the full role of rotation in such stars, and to determine whether other effects ignored in our modeling - e.g., degeneracy and decreasing surface conductivity - also play roles in the dynamo process.

It is a pleasure to thank Gibor Basri, Juri Toomre, A. Sacha Brun, Andrew West, Mark Miesch, and Benjamin Brown for helpful discussions and/or comments on this manuscript. We also gratefully acknowledge Isabelle Baraffe and Gilles Chabrier for supplying the 1D stellar model used here for initial conditions. This work was supported by an NSF Astronomy and Astrophysics postdoctoral fellowship (AST 05-02413). The simulations were carried out with NASA support of Project Columbia, and NSF PACI support of NCSA, SDSC, and PSC.
Babcock, H. W. 1961, ApJ, 133, 572

Barnes, S. A. 2003, ApJ, 586, 464

Biskamp, D. 1993, Nonlinear Magnetohydrodynamics (Cambridge: Cambridge Univ. Press)

Blackman, E. G. 2003a, MNRAS, 344, 707

2003b, in Turbulence and Magnetic Fields in Astrophysics, ed. E. Falgarone \& T. Passot (Berlin: Springer), 614

Blackman, E. G., \& Field, G. B. 2000, ApJ, 534, 984

Boldyrev, S. 2006, Phys Rev. Lett., 96, 115002

Boldyrev, S., \& Cattaneo, F. 2004, Phys. Rev. Lett., 92, 144501

Brandenburg, A., \& Subramanian, K. 2005, Phys. Rep., 417, 1

Browning, M. K., Brun, A. S., \& Toomre, J. 2004, ApJ, 601, 512

Browning, M. K., Miesch, M. S., Brun, A. S., \& Toomre, J. 2006, ApJ, 648, L1 57

Brummell, N. H., Clune, T. L., \& Toomre, J. 2002, ApJ, 570, 825

Brun, A. S., Browning, M. K., \& Toomre, J. 2005, ApJ, 629, 461 (BBT05)

Brun, A. S., Miesch, M. S., \& Toomre, J. 2004, ApJ, 614, 1073 (BMT04)

Brun, A. S., \& Toomre, J. 2002, ApJ, 570, 865

Busse, F. 1970, J. Fluid Mech., 44, 441

Cattaneo, F., \& Hughes, D. W. 2006, J. Fluid Mech., 553, 401

Chabrier, G., Baraffe, I., Allard, F., \& Hauschildt, P. 2000, ApJ, 542, 464

Chabrier, G., Gallardo, J., \& Barrafe, I. 2007, A\&A, 472, L17

Chabrier, G., \& Küker, M. 2006, A\&A, 446, 1027

Charbonneau, P., \& MacGregor, K. B. 1997, ApJ, 486, 502

Childress, S., \& Gilbert, A. D. 1995, Stretch, Twist, Fold: The Fast Dynamo (Berlin: Springer)

Christensen, U. R., \& Aubert, J. 2006, Geophys. J. Int., 166, 97

Clune, T. L., Elliott, J. R., Glatzmaier, G. A., Miesch, M. S., \& Toomre, J. 1999, Parallel Comput., 25, 361

Courvoisier, A., Hughes, D. W., \& Tobias, S. M. 2006, Phys. Rev. Lett. 96, 034503

\section{REFERENCES}

Delfosse, X., Forveille, T., Perrier, C., \& Mayor, M. 1998, A\&A, 331, 581 Dikpati, M., \& Charbonneau, P. 1999, ApJ, 518, 508

Dobler, W., Stix, M., \& Brandenburg, A. 2006, ApJ, 638, 336 (DSB06)

Donati, J. F., Forveille, T., Cameron, A. C., Barnes, J. R., Delfosse, X., Jardine, M. M., \& Valenti, J. A. 2006, Science, 311, 633

Durney, B. R., De Young, B. S., \& Roxburgh, I. W. 1993, Sol. Phys., 145, 207

Durney, B. R., Mihalas, D., \& Robinson, R. D. 1981, PASP, 93, 537

Elliott, J. R., Miesch, M. S., \& Toomre, J. 2000, ApJ, 533, 546

Gilman, P. A. 1976, in IAU Symp. 71, Basic Mechanisms of Solar Activity, ed.

V. Bumba \& J. Kleczek (Dordrecht: Reidel ), 207 1978, Geophys. Astrophys. Fluid Dyn., 11, 157 1979, ApJ, 231, 284

Gilman, P. A., \& Foukal, P. V. 1979, ApJ, 229, 1179

Goldreich, P., \& Sridhar, S. 1995, ApJ, 438, 763

Hawley, S. L., Gizis, J. E., \& Reid, I. N. 1996, AJ, 112, 2799

James, D. J., Jardine, M. M., Jeffries, R. D., Randich, S., Collier Cameron, A., \& Ferreira, M. 2000, MNRAS, 318, 1217

Jones, C. A., \& Roberts, P. H. 2000, J. Fluid Mech., 404, 311

Kitchatinov, L. L., \& Ruediger, G. 1995, A\&A, 299, 446

Knobloch, E., Rosner, R., \& Weiss, N. O. 1981, MNRAS, 197, 45

Küker, M., \& Rüdiger, G. 1999, A\&A, 346, 922

Leighton, R. B. 1969, ApJ, 156, 1

Livermore, P. W., Hughes, D. W., \& Tobias, S. M. 2007, Phys. Fluids, 19, 057101

Miesch, M. S. 1998, Ph.D. thesis, Univ. Colorado, Boulder

Miesch, M. S., Brun, A. S., \& Toomre, J. 2006, ApJ, 641, 618

Miesch, M. S., Elliott, J. R., Toomre, J., Clune, T. L., Glatzmaier, G. A., \& Gilman, P. A. 2000, ApJ, 532, 593

Moffatt, H. K. 1978, Magnetic Field Generation in Electrically Conducting Fluids (Cambridge: Cambridge Univ. Press) 
Moffatt, H. K., \& Proctor, M. R. E. 1982, Geophys. Astrophys. Fluid Dyn., 21, 265

Moffatt, H. K., \& Tsinober, A. 1992, Ann. Rev. Fluid Mech. 24, 281

Mohanty, S., \& Basri, G. 2003, ApJ, 583, 451

Mohanty, S., Basri, G., Shu, F., Allard, F., \& Chabrier, G. 2002, ApJ, 571, 469

Noyes, R. W., Hartmann, L. W., Baliunas, S. L., Duncan, D. K., \& Vaughan, A. H. 1984, ApJ, 279, 763

Olson, P., \& Christensen, U. R. 2006, Earth Planet. Sci. Lett., 250, 561

Ossendrijver, M. 2003, A\&A Rev., 11, 287

Parker, E. N. 1955, ApJ, 122, 293 1975, ApJ, 198, 205 1993, ApJ, 408, 707

Pedlosky, J. 1987, Geophys. Fluid Dyn. (New York: Springer)

Pizzolato, N., Maggio, A., Micela, G., Sciortion, S., \& Ventura, P. 2003, A\&A, 397,147

Pouquet, A., Frisch, U., \& Leorat, J. 1976, J. Fluid Mech., 77, 321

Pouquet, A., \& Patterson, G. S. 1978, J. Fluid Mech., 85, 305

Reiners, A., \& Basri, G. 2007, ApJ, 656, 1121
Reiners, A., et al. 2007, A\&A, 471, L5

Rempel, M. 2006, ApJ, 647, 662

Schekochihin, A. A., Haugen, N. E. L., Brandenburg, A., Cowley, S. C., Maron, J. L., \& McWilliams, J. C. 2005, ApJ, 625, L115

Simon, G. W., et al. 1988, ApJ, 327, 964

Skumanich, A. 1972, ApJ, 171, 565

Sprague, M., Julien, K., Knobloch, E., \& Werne, J. 2006, J. Fluid Mech., 551, 141

Sreenivasan, B., \& Jones, C. 2006, Geophys. J. Int., 164, 467

Steenbeck, M., Krause, F., \& Radler, K. H. 1966, Z. Nat., 21, 369

Stillmach, S., \& Hansen, U. 2004, Phys. Rev. E, 70, 056312:1

Stix, M. 2002, The Sun: an Introduction (Berlin: Springer)

Thompson, C., \& Duncan, R. C. 1993, ApJ, 408, 194

Thompson, M. J., et al. 1996, Science, 272, 1300

Weber, E. J., \& Davis, L. 1967, ApJ, 148, 217

West, A. A., Hawley, S. L., Bochanski, J. J., Covey, K. R., Reid, I. N., Dhital, S., Hilton, E. J., \& Masuda, M. 2007, AJ, submitted

West, A. A., et al. 2004, AJ, 128, 426 